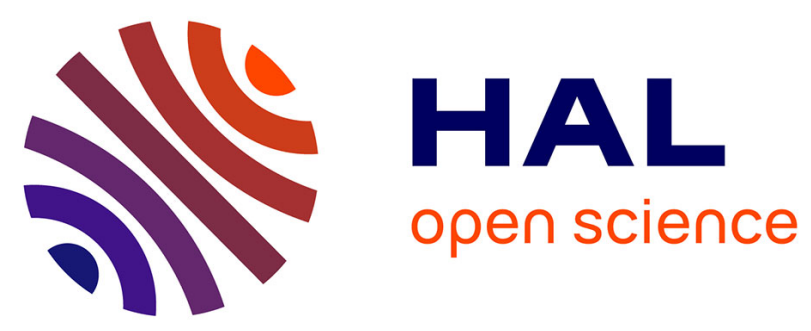

\title{
Structural features of the interaction of MapZ with FtsZ and membranes in Streptococcus pneumoniae
}

Tomáš Hošek, Catherine M Bougault, Jean-Pierre Lavergne, Denis Martinez, Isabel Ayala, Daphna Fenel, Marine Restelli, Cécile Morlot, Birgit Habenstein, Christophe C. Grangeasse, et al.

\section{To cite this version:}

Tomáš Hošek, Catherine M Bougault, Jean-Pierre Lavergne, Denis Martinez, Isabel Ayala, et al.. Structural features of the interaction of MapZ with FtsZ and membranes in Streptococcus pneumoniae. Scientific Reports, 2020, 10 (1), pp.4051. 10.1038/s41598-020-61036-9 . hal-01119769

\section{HAL Id: hal-01119769 \\ https://hal.univ-grenoble-alpes.fr/hal-01119769}

Submitted on 23 Nov 2020

HAL is a multi-disciplinary open access archive for the deposit and dissemination of scientific research documents, whether they are published or not. The documents may come from teaching and research institutions in France or abroad, or from public or private research centers.
L'archive ouverte pluridisciplinaire HAL, est destinée au dépôt et à la diffusion de documents scientifiques de niveau recherche, publiés ou non, émanant des établissements d'enseignement et de recherche français ou étrangers, des laboratoires publics ou privés. 


\title{
SCIENTIFIC REPORTS
}

natureresearch

\section{Structural features of the} interaction of MapZ with FtsZ
and membranes in Streptococcus
pneumoniae

\author{
Tomas Hosek $^{1}$, Catherine M. Bougault ${ }^{1}$, Jean-Pierre Lavergne ${ }^{2}$, Denis Martinez ${ }^{3}$, \\ Isabel Ayala ${ }^{1}$, Daphna Fenel ${ }^{1}$, Marine Restelli ${ }^{2}$, Cecile Morlot ${ }^{1}$, Birgit Habenstein ${ }^{3}$, \\ Christophe Grangeasse ${ }^{2 *}$ \& Jean-Pierre Simorre ${ }^{1^{*}}$
}

Mapz localizes at midcell and acts as a molecular beacon for the positioning of the cell division machinery in the bacterium Streptococcus pneumoniae. MapZ contains a single transmembrane helix that separates the $\mathrm{C}$-terminal extracellular domain from the $\mathrm{N}$-terminal cytoplasmic domain. Only the structure and function of the extracellular domain is known. Here, we demonstrate that large parts of the cytoplasmic domain is intrinsically disordered and that there are two regions (from residues 45 to 68 and 79 to 95) with a tendency to fold into amphipathic helices. We further reveal that these regions interact with the surface of liposomes that mimic the Streptococcus pneumoniae cell membrane. The highly conserved and unfolded $\mathrm{N}$-terminal region (from residues 17 to 43 ) specifically interacts with FtsZ independently of FtsZ polymerization state. Moreover, we show that MapZ phosphorylation at positions Thr67 and Thr68 does not impact the interaction with FtsZ or liposomes. Altogether, we propose a model in which the MapZ-mediated recruitment of FtsZ to mid-cell is modulated through competition of MapZ binding to the cell membrane. The molecular interplay between the components of this tripartite complex could represent a key step toward the complete assembly of the divisome.

The initiation of bacterial cell division requires the identification of the cell's midpoint prior to the assembly of a highly dynamic protein complex, called the divisome ${ }^{1}$. The divisome constricts the cell and assembles the cell wall depending on the tubulin-like protein $\mathrm{FtsZ}^{2}$. FtsZ polymerizes into protofilaments that ultimately assemble as bundles or clusters, forming a highly dynamic ring called the Z-ring ${ }^{3}$. Treadmilling of FtsZ filaments, a process that is fine-tuned by several accessory proteins, allows for cell constriction together with the assembly of the septal cell wall, giving rise to the two newborn cells. Therefore, a key event for successful division of the bacterial cell is the selection of the site of cell division and the proper positioning of FtsZ.

The positioning of the Z-ring at the cell center should not interfere with the replication and segregation of the chromosome. In Escherichia coli and Bacillus subtilis, the nucleoid occlusion system (NO) ${ }^{4}$ and the Min system ${ }^{5}$ allows the assembly of the Z-ring at mid-cell only when chromosome replication and segregation are completed ${ }^{6}$. However, some bacteria use other mechanisms to position the Z-ring at the cell center. For instance, Z-ring positioning is governed by the protein MipZ in the bacterium Caulobacter crescentus ${ }^{7}$. This protein is conserved in gamma-proteobacteria and also prevents the assembly of Z-ring near the cell poles. By contrast, bacteria like Streptomyces coelicolor ${ }^{8}$ and Myxococcus xanthus $^{9}$ employ alternative mechanisms unrelated to the NO, Min, and MipZ systems. SsgAB in S. coelicolor and PomZ in M. xanthus localize to the mid-cell prior to FtsZ, where they promote FtsZ polymerization and positively drive the assembly of the Z-ring. However, it still remains unclear how these two systems localize at mid-cell.

In the human pathogen Streptococus pneumoniae, the mid-cell positioning of FtsZ is proposed to rely on chromosome segregation ${ }^{10}$ and the MapZ protein (alternately termed LocZ) ) $^{11,12}$. S. pneumoniae is an ovoid-shaped bacterium that maintains its characteristic cell shape by the coordinated peptidoglycan (PG) assembly at mid-cell,

${ }^{1}$ Univ. Grenoble Alpes, CNRS, CEA, Institut de Biologie Structurale, F-38000, Grenoble, France. ${ }^{2}$ Molecular Microbiology and Structural Biochemistry, CNRS UMR 5086, Université de Lyon, Lyon, France. ${ }^{3}$ Institute of Chemistry and Biology of Membranes and Nano-objects, CBMN-CNRS Université de Bordeaux, Pessac, France. *email: c.grangeasse@ibcp.fr; jean-pierre.simorre@ibs.fr 
producing the new cell-hemispheres in between the old hemispheres ${ }^{13}$. Consequently, the boundary between new and old cell wall material (i.e. the cell equator) moves away from mid-cell as the cell elongates ${ }^{14}$. At the beginning of the cell cycle, MapZ forms a stable ring structure at mid-cell, which is proposed to act as an anchor to guide FtsZ treadmilling ${ }^{11,15}$. Just before constriction begins, the MapZ ring splits into two rings which move apart during cell growth to eventually mark the future cell division sites of the two daughter cells. MapZ is a membrane protein with a single transmembrane helix separating a cytoplasmic amino-terminal domain and an extracellular carboxy-terminal domain. The structure of the extracellular domain of MapZ shows a bi-modular organization with two structured subdomains separated by a flexible linker ${ }^{16}$. The membrane proximal-domain serves as a pedestal for the membrane distal C-terminal domain, which interacts with PG. The interaction with PG allows the two MapZ rings to separate at the speed of cell elongation. The extracellular domain of MapZ thus allows the protein to position at the future division sites, while the $\mathrm{N}$-terminal domain guides FtsZ positioning through direct interaction. Little information is available concerning the structural organization of the cytoplasmic domain of MapZ and its interaction with FtsZ ${ }^{15,17}$. It was reported that the N-terminal region of MapZ, from residue 1 to 41, is required for the direct interaction with FtsZ ${ }^{11}$. In addition, the cytoplasmic domain of MapZ can be phosphorylated on two distinct threonine residues (T67 and T78) by StkP, a serine/threonine protein-kinase crucial for cell division and morphogenesiss ${ }^{18}$. While MapZ phosphorylation seems to be crucial for cell constriction, it has no impact on the positioning of FtsZ at mid-cell, suggesting that MapZ could be involved in cell division at two temporally distinct steps.

To gain structural knowledge about the cytoplasmic domain of MapZ and to decipher the details of its interaction with FtsZ, we used several Nuclear Magnetic Resonance (NMR) spectroscopy approaches. We show that the domain is intrinsically highly disordered. We demonstrate that the N-terminal domain of MapZ interacts with FtsZ monomers and protofilaments as well as with negatively charged lipids, suggesting that this region also interacts with the cell membrane. We further provide evidence that the acyl chain of lipids are more mobile in the presence of $M a p Z_{c y t o}$, suggesting that the interaction between MapZ and the membrane influences the recruitment of FtsZ and the assembly of the Z-ring at mid-cell. Last, we found that phosphorylation of MapZ $Z_{c y t o}$ does not significantly affect these interactions. Altogether, this work provides detailed structural insights toward the ultimate understanding of the role of the MapZ in the cell division of S. pneumoniae.

\section{Results and Discussion}

MapZ intracellular domain is intrinsically disordered. The intracellular domain of MapZ (MapZ stretches from residues 1 to 159 and can be phosphorylated at threonine 67 and 78 by the serine/threonine kinase StkP (Fig. 1a) ${ }^{11,12}$. To assess the structural organization of this domain (see amino acid sequence in Fig. 2a), we first overproduced and purified ${ }^{15} \mathrm{~N}$-labeled MapZ $Z_{\text {cyto }}$. The ${ }^{1} \mathrm{H}_{-}{ }^{15} \mathrm{~N}$ correlation spectrum of this MapZ $\mathrm{Z}_{\text {cyto }}$ construct displays typical features of an unfolded protein, i.e. intense narrow peaks and very low chemical shift dispersion, especially in the proton dimension (Fig. 1b). This observed unfolded state confirms the disorder score predicted from the primary sequence by the IUPred software ${ }^{19}$ (Fig. 2b). Indeed, the disorder score obtained from IUPred is 0.8 , characteristic of an unfolded domain, except for residues 22 to 86 , where the score is closer to 0.5.

The cytoplasmic domain of MapZ therefore appears to be mostly an intrinsically disordered region (IDR) with moderate tendency to be structured for the amino acid region 22 to 86 . Such regions with predicted low-score disorder frequently contain "molecular recognition features" (MoRFs) that consist of an amino-acid stretch that either gains stable fold upon binding to its partner, or stays unstructured and rapidly probes the surface of the interaction partner while simultaneously switching between multiple low-affinity binding sites ${ }^{20-22}$. The possible participation of the N-terminal region of MapZ in protein-protein interactions is supported by the presence of a large patch of conserved amino acids extending from residues 18 to 67 , as emphasized by the Consurf amino acid conservation scores (Figs. 2a and S1) obtained from multiple sequence alignment of MapZ proteins from different Streptococcaceae ${ }^{23}$.

Structural recognition features in $\mathrm{MapZ}_{\text {cyto }}$. To further characterize the folding propensity of residues 22 to 86 , backbone resonances were assigned using the standard set of $3 \mathrm{D}{ }^{1} \mathrm{H},{ }^{13} \mathrm{C},{ }^{15} \mathrm{~N}$-multidimensional NMR experiments ${ }^{24}$. With a more than $95 \%$ completion of backbone resonance assignment we could calculate chemical shift deviations from neighbor-corrected random coil values (extracted from the intrinsically disordered protein library ${ }^{25}$ ) for most residues and structural propensities could be deduced from these experimental data ${ }^{26}$. Resulting neighbor-corrected structural propensity ( $\mathrm{ncSP}$ ) values of $\mathrm{MapZ}_{c y t o}$ are reported in Fig. $2 \mathrm{c}$ for each residue along the protein sequence.

To identify marked transient secondary structure, we looked for amino acid stretches with ncSP values above 0.25 and found three regions with helical folding propensity. Regions spanning residues 45 to 68,79 to 95 , and 142 to 156 showed ncSP values comprised between the threshold value and $0.36,0.42$, and 0.33 , respectively. We screened these regions with the Heliquest server ${ }^{27}$ to search for potential hydrophobic patches. The first region, which contains the conserved residues 45 to 68 (Figs. 2a and S1), presents $\pi$-helix conformation with a face containing mainly charged residues (D46/D50/K54/E59/D63) opposite to a hydrophobic face composed of non-polar residues I48/Y52/H56/I60/F65, emphasizing its potential as an interaction interface. The second region with $\alpha$-helical propensity (residues 79 to 95 ) was not predicted by the disorder score in Fig. $2 \mathrm{~b}$ and encompasses residues that are less conserved (Fig. 2a). It contains a similar distribution of residues with a hydrophobic patch with the residues L81/I85/M88/V92 on the opposite face to a highly charged face with residues D83/E87/E90/K94. In contrast, no hydrophobic face emerges from the third region with helical propensity (residues 142 to 156), making this region a weaker candidate for molecular interaction compared to the previous two.

We also measured ${ }^{15} \mathrm{~N}$-relaxation properties of $\mathrm{MapZ}_{\text {cyto }}$ backbone amide groups. The average values of the $\mathrm{R}_{1}$ and $\mathrm{R}_{2}$ relaxation rate constants were $1.7 \pm 0.1 s^{-1}$ and $7 \pm 2 s^{-1}$, respectively. As well, the average ${ }^{1} \mathrm{H}^{-15} \mathrm{~N}$ heteronuclear nOe was below 0.5 across the cytoplasmic domain of MapZ, which is typical for IDRs of similar size 


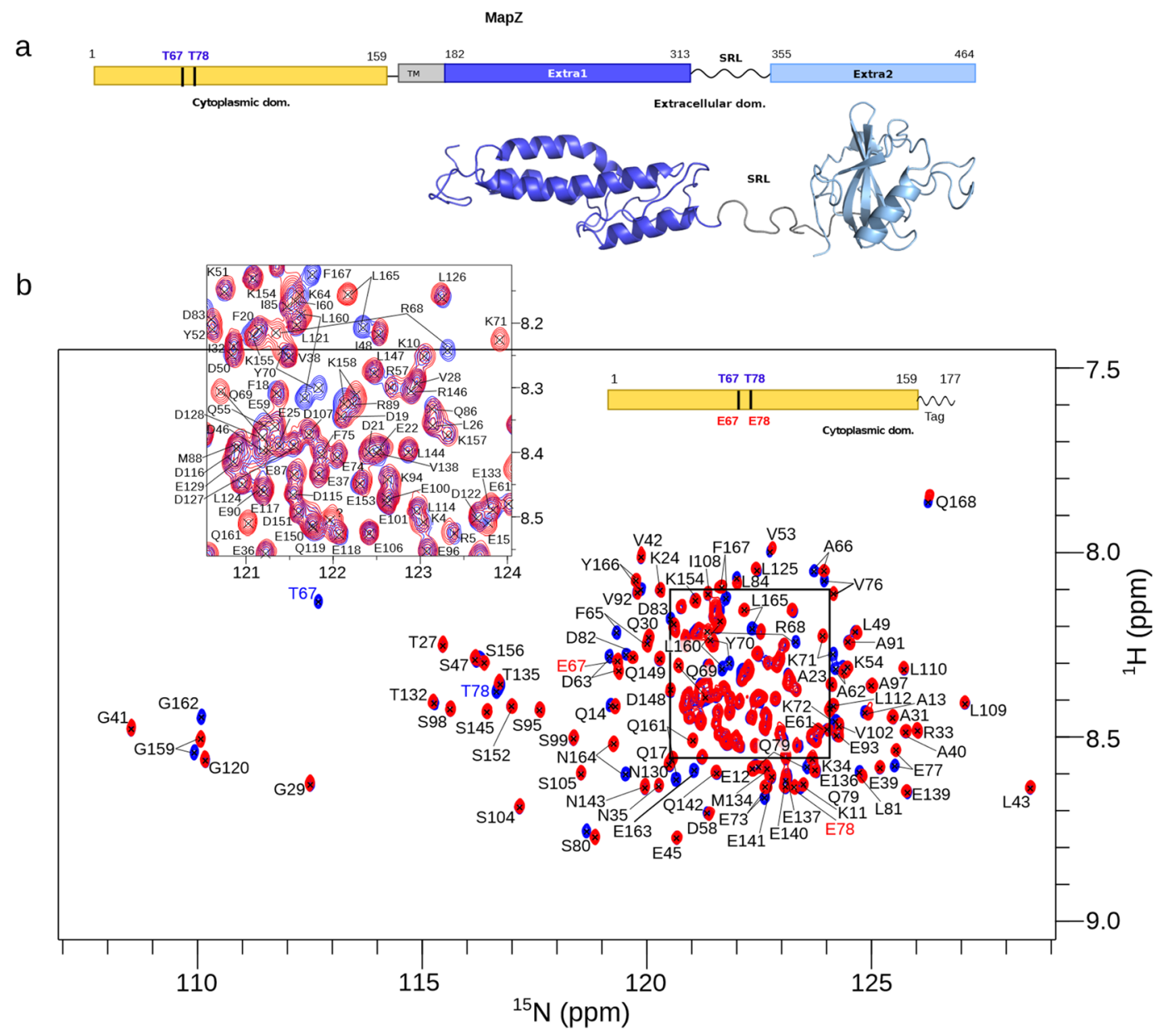

Figure 1. Structural features of MapZ from S. pneumoniae. (a) Domain organization of the full-length MapZ protein of $S$. pneumoniae. The protein consists of a cytoplasmic domain of 159 residues (yellow), linked by a transmembrane helix (grey) to an extracellular domain of 282 residues. Tertiary structure of this extra-cellular domain has been solved ${ }^{16}$ and reveals two structurally independent domains (dark and light blue) linked by a flexible Serine-Rich Linker (SRL). (b) Overlay of the ${ }^{1} \mathrm{H}-{ }^{15} \mathrm{~N}$ BEST-TROSY spectra recorded for ${ }^{13} \mathrm{C},{ }^{15} \mathrm{~N}$ labeled wild-type $\mathrm{MapZ}_{\text {cyto }}$ (blue) and $\mathrm{MapZ}_{\text {cyto }}{ }^{2 T E}$ phosphomimetic construct (T67E/T78E MapZ $\mathrm{Z}_{\text {cyto }}$ ) (red). Protein samples were prepared at $0.2 \mathrm{mM}$ in $30 \mathrm{mM}$ HEPES, $200 \mathrm{mM} \mathrm{KCl}$ buffer at pH 7.5. Both $2 \mathrm{D}{ }^{1} \mathrm{H}-{ }^{15} \mathrm{~N}$ experiments were collected on a 16.5 -T spectrometer at $5{ }^{\circ} \mathrm{C}$. Assignments of amide resonances for MapZ $\mathrm{Z}_{\text {cyto }}$ and $\mathrm{MapZ}_{\text {cyto }}{ }^{2 T E}$ are reported in black, the mutated residues 67 and 78 are indicated in blue and red for wild-type and phosphomimetic constructs, respectively. Only minimal chemical shift differences are observed between these spectra and shifts in the cytoplasmic domain correspond solely to residues neighboring the mutated sites. In both protein constructs, residues 1 to 159 belong to the MapZ sequence, while residues 160 to 168 are reminiscent of the His-TEV site tag after TEV cleavage.

at $5{ }^{\circ} \mathrm{C}$ (Fig. S2) ${ }^{28}$. Interestingly, $\mathrm{R}_{2} / \mathrm{R}_{1}$ ratios remain relatively constant along the sequence except for regions spanning from residues 45 to 68 and 79 to 95 , which were previously identified with modest but marked helical propensity (Fig. $2 \mathrm{~d}$ ). This relaxation heterogeneity is due to an increase in the $\mathrm{R}_{2}$ rate constant that could result from differences in sequential $\tau_{c}$ and the presence of a transient local compactness. Combined with the helical propensity of these two regions (Fig. 2c), this strongly supports the existence of transitory amphipathic helical secondary structures in $\mathrm{MapZ}_{\text {cyto }}$ that could promote interaction with cytoplasmic partners.

Altogether, the chemical shift and ${ }^{15} \mathrm{~N}$-relaxation parameters identified two regions of local compactness with higher $\alpha$-helical propensity for residues 45 to 68 and 79 to 95, respectively. The first region displays a patch of highly conserved residues (Figs. $2 \mathrm{a}$ and $\mathrm{S} 1$ ), whereas both regions display a hydrophobic face opposite to a highly charged face. The high local mobility of $\mathrm{MapZ}_{\text {cyto }}$ domain, combined with the presence of these two more compact short domains, grant it an advantage in terms of interaction with possible binding partners, notably FtsZ, which is the only known protein partner of MapZ ${ }^{11}$.

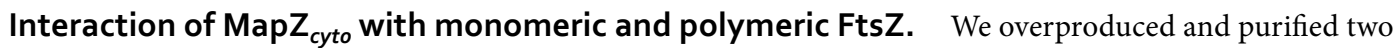
different versions of recombinant FtsZ (see Methods section). The first version, Fts $Z_{a}$, corresponded to the wild-type FtsZ from S. pneumoniae (strain ATCC BAA-255/R6) with no purification tag and was produced in 

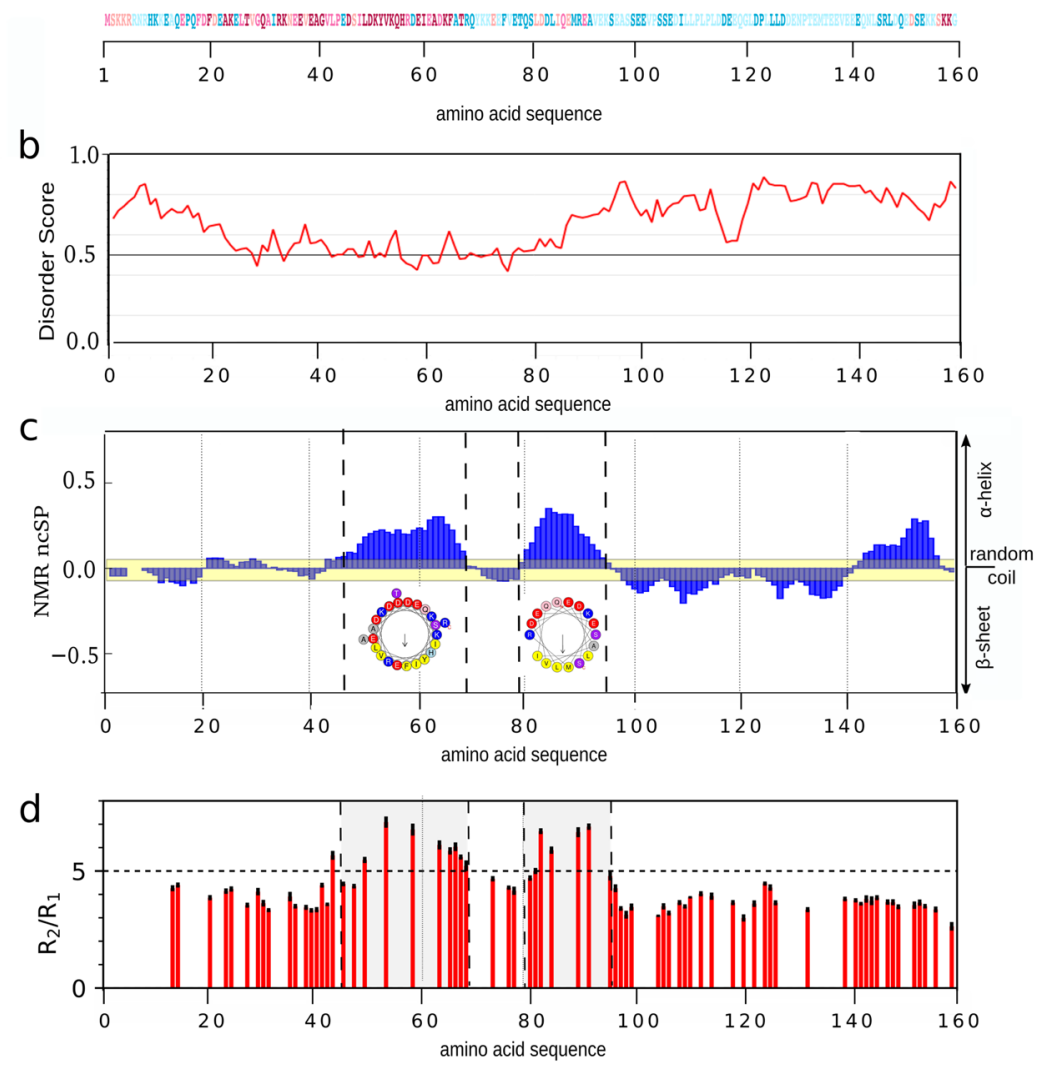

Figure 2. Structural organization of MapZ cytoplasmic domain (residues 1-159). (a) Amino acid conservation scores as calculated by the Consurf webserver (http://consurf.tau.ac.il/) ${ }^{23}$ are displayed on the MapZ $\mathrm{Z}_{\text {cyto }}$ protein sequence. Scores range from 0 (not conserved, white) to 9 (highly conserved, magenta). (b) Disorder scores predicted along the protein sequence by the IUPred software (http://iupred2a.elte.hu/) ${ }^{19}$. Highly disordered regions correspond to scores above 0.5 . (c) Neighbor-corrected structural propensity (ncSP) $)^{25}$ scores calculated from C', $C^{\alpha}$, and $C^{\beta}$ NMR chemical shifts of MapZ $Z_{\text {cyto }}$. ncSP scores reveal the propensity to form secondary structures (zero for random coil, positive for $\alpha$-helices and negative for $\beta$-sheets). The yellow band represents a cutoff value of 0.1 , which outlines the absence of marked structural propensity. A helix plot representation obtained from the HELIQUEST server ${ }^{27}$ is displayed for two regions ( $\pi$-helix for residues 45 to 68 and $\alpha$-helix for residues 79 to 95) with larger helical propensity. Positively-charged, negatively-charged, polar, and non-polar hydrophobic residues are colored in dark-blue, red, purple, and yellow, respectively. (d) Ratio of $R_{2} / R_{1}$ relaxation rate constant values (in red) and corresponding errors (in black) obtained at $5{ }^{\circ} \mathrm{C}$ displayed on the MapZ $\mathrm{Z}_{\text {cyto }}$ sequence. $R_{2} / R_{1}$ ratio values significantly above average are identified for regions spanning residues 45 to 68 and 79 to 95 . This might suggest the presence of a transient local compactness for those two regions.

E. coli $\mathrm{C} 41(\mathrm{DE} 3)$. The $\mathrm{FtsZ}_{b}$ construct was produced in E. coli BL21 (DE3) and expressed from a pGEX vector encoding the glutathione S-transferase (GST)-tagged protein, whereby the N-terminal GST tag was removed by Tobacco Etch Virus (TEV) protease. The only difference between the two constructs was a glycine residue at the $\mathrm{N}$-terminus coming from the tag scar in $\mathrm{FtsZ}_{b} .{ }^{1} \mathrm{H}$ NMR spectra collected on both samples at $25^{\circ} \mathrm{C}$ in the same buffer suggest that both protein constructs have the same structure (Fig. S3a).

We first investigated the interaction between $\mathrm{MapZ}_{c y t o}$ and monomeric FtsZ, i.e. in the absence of guanosine triphosphate (GTP) and $\mathrm{MgCl}_{2}$. For this purpose, we recorded ${ }^{1} \mathrm{H},{ }^{15} \mathrm{~N}$ BEST-TROSY spectra on ${ }^{15} \mathrm{~N}$-labeled MapZ $Z_{c y t o}$ alone or in the presence of increasing amount of unlabeled, monomeric FtsZ (up to 6-fold molar excess). Due to the tendency of FtsZ to form oligomers/bundles and polymerize at higher concentration, we used a MapZ $Z_{\text {cyto }}$ protein concentrations below $90 \mu \mathrm{M}$, typically in the range of 30 to $50 \mu \mathrm{M}$. Samples used for different titration points were prepared from the same protein and buffer batches and contained the same concentration of MapZ $Z_{c y t o}$. An example of MapZ $Z_{c y t o}$ BEST-TROSY spectra collected before and after addition of 4.7 molar equivalent of Fts $Z_{a}$ is shown in Fig. 3a. Strikingly, addition of monomeric FtsZ caused no chemical shift perturbation but a decrease in the intensity of some of signals. For further analysis, the decrease in signal intensity was quantified for each of the amide resonances and the values are reported along the MapZ $\mathrm{Cyto}_{\text {sto }}$ sequence (Fig. $3 \mathrm{~b}$ ). The $\mathrm{N}$-terminal residues 17 to 43 experienced an intensity decrease of up to $70 \%$ for the $\mathrm{MapZ}_{c y t o}: \mathrm{FtsZ}_{a} 1: 4.7$ ratio, whereas other residues of the cytoplasmic domain of MapZ remained largely unaffected. Similar results were obtained with the Fts $Z_{b}$ construct in the presence of which the intensity of the same signals decreased stronger at higher MapZ $Z_{c y t o}: F_{s} Z_{b}$ protein ratios (Fig. S4a). These results suggest that the 17-to-43 N-terminal region of 


\section{a}
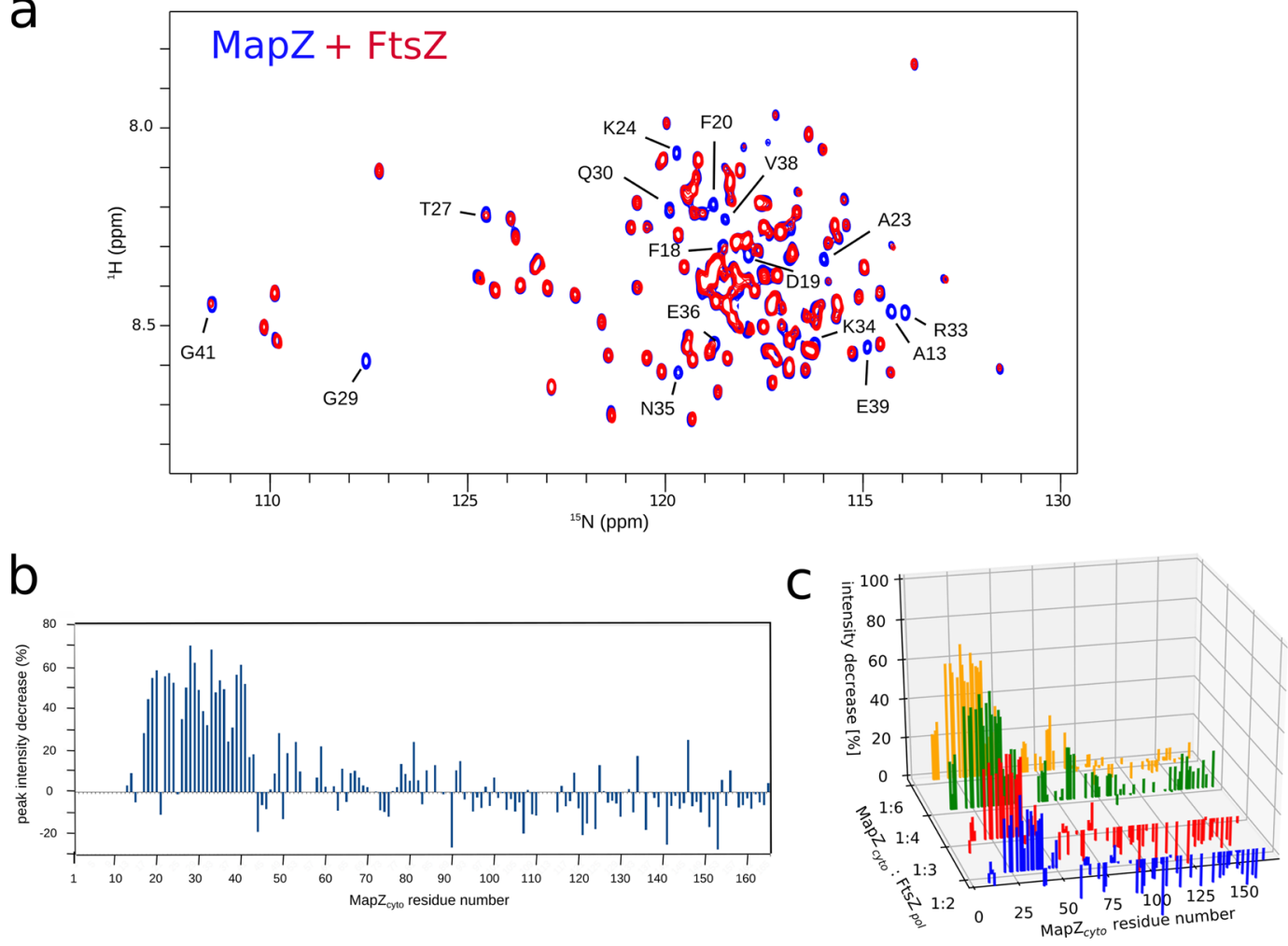

Figure 3. Interaction of $\mathrm{MapZ}_{\text {cyto }}$ with monomeric FtsZ. (a) Superimposition of the ${ }^{1} \mathrm{H}-{ }^{15} \mathrm{~N}$ BEST-TROSY correlation spectra recorded on $\mathrm{MapZ}_{c y t o}$ in absence (blue) and in presence (red) of 4.7 molar equivalent of FtsZ $Z_{a}$. The samples were prepared with $88 \mu \mathrm{M}$ final concentration of ${ }^{15} \mathrm{~N} \mathrm{MapZ} Z_{c y t o}$ in $30 \mathrm{mM} \mathrm{HEPES}, 50 \mathrm{mM} \mathrm{KC}$ buffer at pH 7.5. NMR experiments were recorded on 16.5-T Bruker Avance III spectrometer at $5^{\circ} \mathrm{C}$. (b) Peak intensity decrease upon addition of 4.7 molar equivalent of $\mathrm{FtsZ}_{a}$ on $\mathrm{MapZ}_{c y t o}$ are reported as a function of the residue position in MapZ primary sequence. For each assigned resonance that is not significantly overlapped, the intensities $\mathrm{I}_{0}$ and $\mathrm{I}_{1}$ are measured in the reference blue spectrum containing only $\mathrm{MapZ}_{c y t o}$ and in the red spectrum containing the $\mathrm{MapZ}_{c y t o}$-FtsZ $\mathrm{Z}_{a}$ mixture, respectively, reported in (a). The peak intensity decrease (in $\%$ ) is calculated as the $\frac{\mathrm{I}_{0}-\mathrm{I}_{1}}{\mathrm{I}_{0}}$ ratio. (c) A $3 \mathrm{D}$ plot reporting the peak intensity decrease as a function of the residue position in MapZ primary sequence along $\mathrm{MapZ}_{c y t o}$ titration by polymeric FtsZ $\mathrm{F}_{b}\left(\mathrm{FtsZ}_{b}\right)$. Blue, red, green and yellow histograms represent 1:1, 1:3, 1:4 and 1:6 molar ratios of $\mathrm{MapZ}_{\text {cyto }}:$ Fts $Z_{p o l}$. Samples were prepared from $0.5 \mathrm{mM}$ and $210 \mu \mathrm{M}$ stock solutions of $\mathrm{MapZ}_{c y t o}$ and FtsZ $\mathrm{pH}$ 7.5. The final MapZ $\mathrm{Z}_{\text {cyto }}$ concentration in each sample was $30 \mu \mathrm{M}$, and $10 \mathrm{mM} \mathrm{GTP}$ and $\mathrm{MgCl}_{2}$ were added to polymerize FtsZ, as verified by electron microscopy.

$\mathrm{MapZ}_{\text {cyto }}$ interacts with monomeric FtsZ, whereas the remainder of the cytosolic region stays completely flexible in the protein-protein complex. This conclusion differs from recently reported data by Feng et al. ${ }^{17}$, who proposed several polar residues to be involved in the MapZ-FtsZ interaction. However, the chemical shifts of these residues, which are distributed all along the amino acid sequence, are more likely induced by small variations in small ionic strength of the buffer, or residual magnesium ions coming from the FtsZ preparation. Our conclusion is consistent with our previous observations that MapZ alleles lacking the first $42 \mathrm{~N}$-terminal residues were unable to promote correct FtsZ placement ${ }^{11}$.

We then turned to polymeric FtsZ considering that its interaction with $\mathrm{MapZ}_{\text {cyto }}$ might be dependent on the oligomeric state of FtsZ. We thus collected ${ }^{1} \mathrm{H},{ }^{15} \mathrm{~N}$ BEST-TROSY spectra after addition of GTP and $\mathrm{MgCl}_{2}$ to $\mathrm{MapZ}_{c y t o}:$ FtsZ mixtures at different molar ratios. Parallel samples were analyzed by negative-staining electron microscopy to ascertain FtsZ polymerization under the conditions used (Fig. S3b). Again, no chemical shift perturbation was observed, but resonance intensity decreased for some of the amide resonances. Data are reported in Fig. 3b for MapZcyto:FtsZ $Z_{a}$ and in Fig. $3 c$ for different MapZ $Z_{c y t o}: F_{s t} Z_{b}$ mixtures. The intensity changes along the MapZ sequence was similar compared to those observed for the monomeric form of FtsZ, suggesting that the interaction site of $\mathrm{MapZ}_{c y t o}$ with monomeric or oligomeric FtsZ was essentially the same. In both cases, the overall decrease in signal intensity as a function of the $\mathrm{MapZ}_{\text {cyto }}$ :FtsZ ratios suggested apparent equilibrium dissociation constants in the range of $10-100 \mu \mathrm{M}$ if one considers a 1:1 complex (which was two orders of magnitude weaker than the value reported by Feng et al. ${ }^{17}$ ). The apparent dissociation constant was slightly lower for the FtsZ oligomers than for the monomers (Fig. S4) and depended on the salt concentration.

To further investigate the interaction between polymeric FtsZ and $\mathrm{MapZ}_{c y t o}$, we determined the capacity of FtsZ to form filament bundles in the presence of $\mathrm{MapZ}_{\text {cyto }}$ by negative-stain electron microscopy. In a polymerization buffer containing $8 \%$ polyvinyl alcohol (PVA), FtsZ $_{a}$ filaments formed bundles that were longer than $10 \mu \mathrm{m}$ 

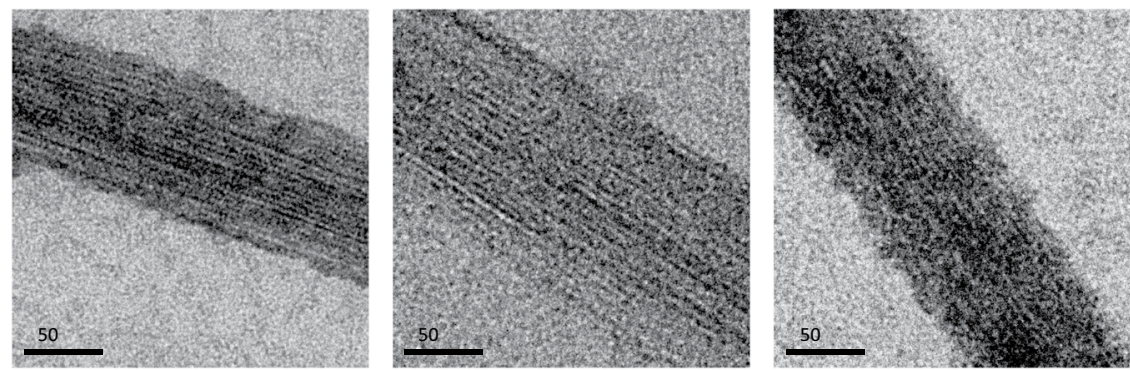

Figure 4. Effect of $\mathrm{MapZ}_{\text {cyto }}$ on FtsZ bundling. Negative-stain electron microscopy images of FtsZ filament bundles imaged with a calibrated nominal magnification of 23,000. FtsZ polymerization and filament bundling were performed for $15 \mathrm{~min}$ at room temperature in buffer at $\mathrm{pH} 7.6$ containing $50 \mathrm{mM}$ HEPES, $200 \mathrm{mM} \mathrm{KCl}$, $5 \mathrm{mM} \mathrm{MgCl}_{2}, 5 \mathrm{mM} \mathrm{GTP}$, and $8 \%$ (wt/vol) PVA, in the absence (left panel) or in the presence of $50 \mu \mathrm{M} \mathrm{MapZ}_{c y t o}$ (middle panel) or $50 \mu \mathrm{M}$ BSA (right panel).

and between 50 to $150 \mathrm{~nm}$ thick (Figs. 4 and S5). MapZ ${ }_{c y t o}$ at an equimolar ratio relative to FtsZ had no effect on FtsZ bundles although the protein concentrations were 500 -fold above the apparent dissociation constant of the FtsZ-MapZ interaction ${ }^{11}$. When MapZ $\mathrm{Cyto}_{\text {and }}$ and Z were mixed at a 10:1 ratio, there was no major effect on the FtsZ bundles at high magnification (Fig. 4), but FtsZ bundles and isolated FtsZ filaments seemed less abundant (Fig. S5), consistent with a previous study by Feng et al. who reported that the addition of MapZ destabilizes the bundling of FtsZ polymers ${ }^{17}$. However, we observed a similar destabilization effect when bovine serum albumine (BSA) was used instead of MapZ (Figs. 4 and S5). On the other hand, in the absence of PVA, an equimolar or 10:1 $\mathrm{MapZ}_{\text {cyto }}:$ FtsZ ratio did not promote FtsZ bundling. Altogether, these observations suggest that the formation of stable FtsZ filaments and their bundling are affected by high protein concentrations, but are not specifically affected by the presence of MapZ $Z_{c y t o}$. However, we cannot exclude that the modulation of FtsZ filament formation and/or bundling by MapZ might be enhanced by the presence of a yet unknown protein partner.

Our NMR and EM data show that a relatively low affinity complex forms between $\mathrm{MapZ}_{c y t o}$ and monomeric as well as polymeric FtsZ. Only the 17-to-43 residue stretch at the N-terminus of MapZ interacts with FtsZ. This highly conserved region contains MoRFs that remain largely unstructured and rapidly probe the surface of its FtsZ binding partner. This functional role of MapZ could be crucial, as the complex needs to be formed transiently at different stages of the division. This hypothesis is also consistent with the findings that MapZ serves both for initial positioning of FtsZ at mid-cell, suggesting an interaction with FtsZ monomers, and for the regulation of the Z-ring constriction, suggesting an interaction with FtsZ bundles ${ }^{11}$.

Interaction of MapZ $\mathrm{Z}_{c y t o}$ with liposomes that mimic the cell membrane of S. pneumoniae. Since $\mathrm{MapZ}_{c y t o}$ domain is an intrinsically disordered domain that is anchored to the cell membrane in the context of the full-length protein, we investigated its capacity to interact with a membrane-like environment. For this purpose, we first considered small unilamelar vesicles (SUVs) with a lipid composition that is similar to the composition of the cytoplasmic membrane of $S$. pneumoniae. These monodisperse 50-nm wide liposomes consisted of a mixture of two lipids with negatively charged polar-heads, 1-palmitoyl-2-oleoyl-sn-glycero-3-phospho-(1'-rac-glycerol) (POPG) and cardiolipin (CL), mixed in a 1:1 molar ratio ${ }^{29}$. Their interaction with $\mathrm{MapZ}_{\text {cyto }}$ was studied using NMR spectroscopy and ${ }^{1} \mathrm{H}-{ }^{15} \mathrm{~N}$ BEST-TROSY correlation spectra of ${ }^{15} \mathrm{~N}$ - MapZ $\mathrm{Z}_{\text {cyto }}$ were recorded after addition of different molar ratio of SUVs (one SUV contains approximately 7000 lipid molecules). Consistent with previously described experiments on the interaction between $\mathrm{MapZ}_{\text {cyto }}$ and FtsZ, neither new signals nor chemical shift perturbations of existing resonances were observed for a 1:62 MapZ $Z_{c y t o}$ :lipid molar ratio (Fig. 5a). Instead, some of the resonances showed decreased intensity after addition of liposomes to MapZ $Z_{c y t o}$ (Fig. 5a,b). This decrease even exacerbated with the addition of higher concentration of liposome (Fig. 5c) and the affected residues localized at the N-terminus of the cytoplasmic domain of MapZ, as shown in Fig. 5b,c, demonstrating a clear interaction between the lipids and the protein.

Complex formation between $\mathrm{MapZ}_{c y t o}$ and SUVs was further confirmed by DOSY NMR experiments (see Supplementary Information). Indeed, this type of experiment provides an estimate for the translational diffusion coefficient of the various components of biological samples, as this coefficient is sensitive to differences in molecular weight. We focused on the resolved methyl protons of the protein and used these to determine the diffusion coefficient along the titration of $\mathrm{MapZ}_{\text {cyto }}$ with SUVs. As shown in Fig. S6, we observed a decrease in the diffusion coefficient D with increasing concentrations of SUVs in the sample (the value of D diminished by a factor up to 3 for a MapZ:lipid molar ratio of 1:62). This observation is consistent with the formation of a protein-SUV complex in rapid exchange, i.e. a rapid interconversion between the free and complexed protein forms.

When intensity perturbations were analyzed in more detail at a high lipid to MapZ $\mathrm{Z}_{\text {cyto }}$ ratio (Fig. 5b), residues 11 to 110 show values of the intensity decrease that are above $20 \%$, with lower values towards the end of this region. This result suggests that, in the presence of SUVs, the C-terminal part of MapZ $Z_{c y t o}$ (residues 110 to 159) remains flexible upon interaction of the $\mathrm{N}$-terminal part (residues 11 to 110) with the liposomes. Interestingly, this N-terminal region contains the two previously identified portions with helical propensities (residues 45 to 68 and 79 to 95 (Fig. 2c)). Interaction between MapZ $Z_{c y t o}$ and liposomes could thus be mediated by the formation of these amphipathic helices, the first of them being highly conserved in MapZ proteins of all Streptococcaceae. The $\mathrm{N}$-terminal region of $\mathrm{MapZ}_{c y t o}$ is also characterized by the presence of positively charged amino acids (residues 
a

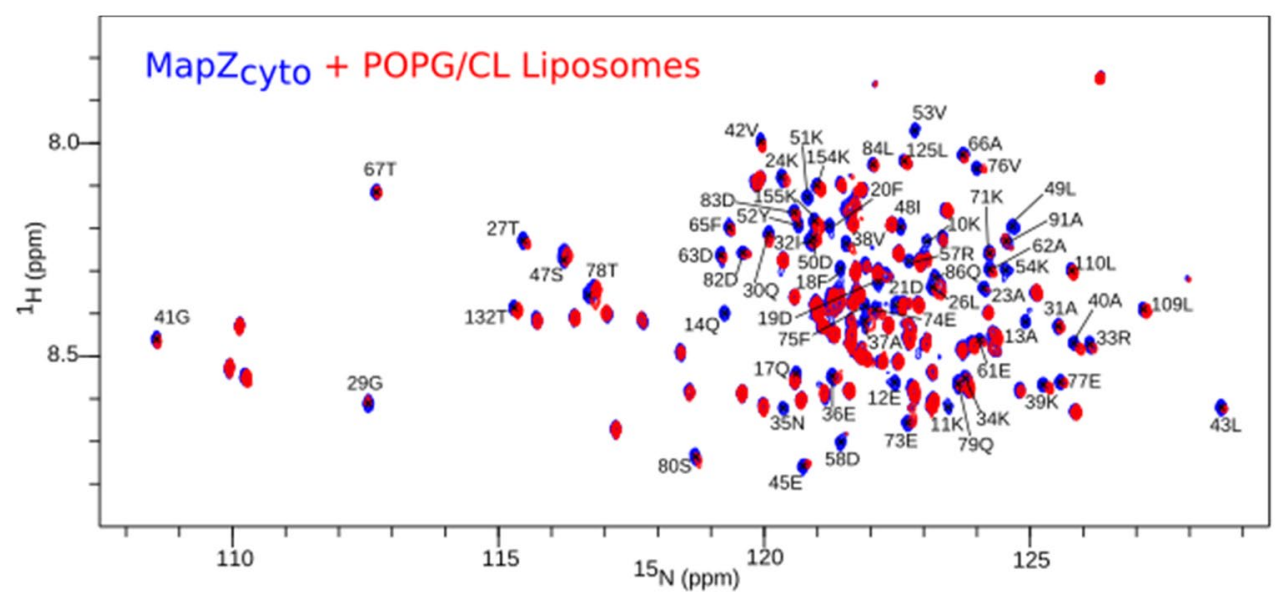

b

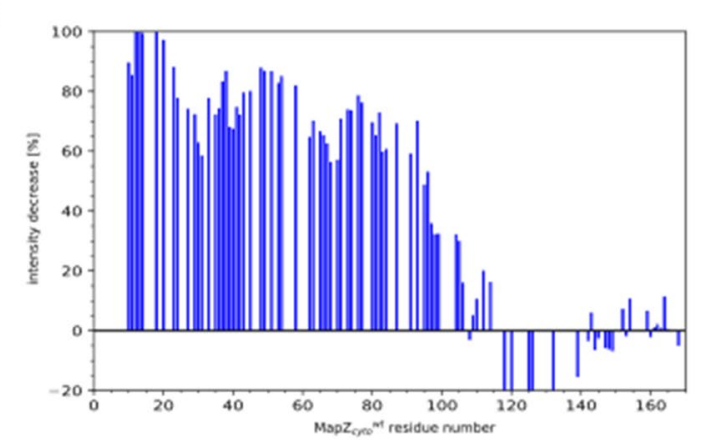

C

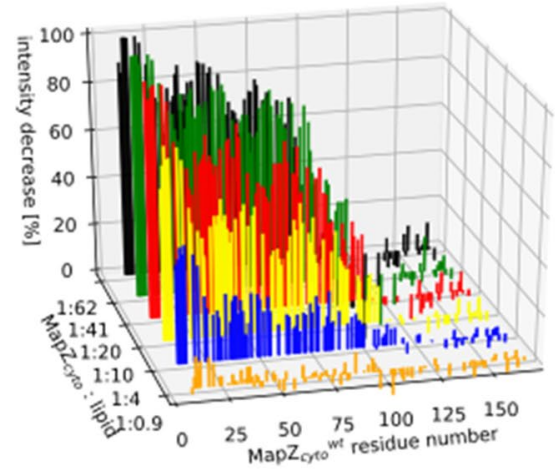

d

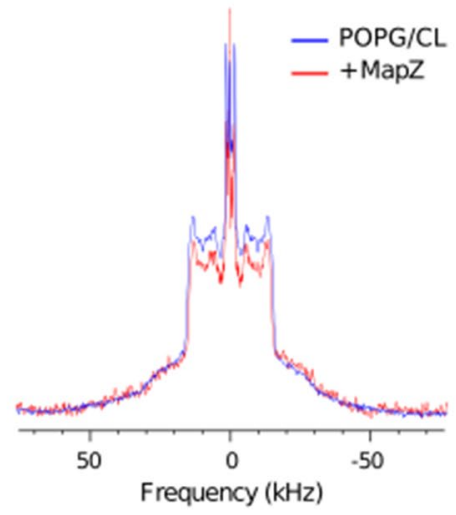

e

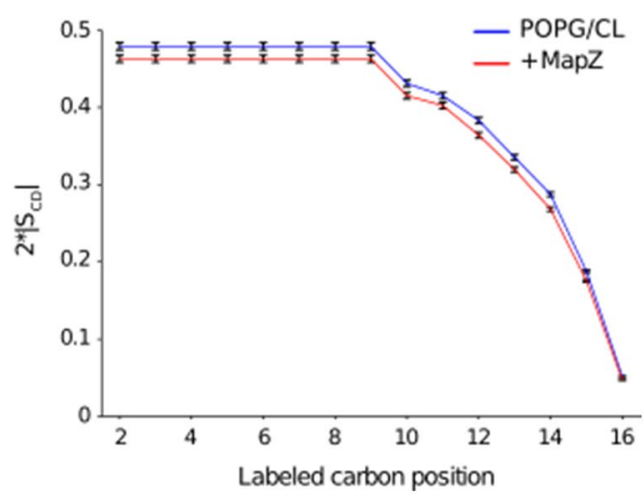

Figure 5. Interaction between MapZ $Z_{c y t o}$ and liposomes. (a) ${ }^{1} \mathrm{H}-{ }^{15} \mathrm{~N}$ BEST-TROSY spectra of ${ }^{15} \mathrm{~N}$-labeled MapZ $\mathrm{Z}_{\text {cyto }}$ recorded in the absence (blue) and in the presence (red) of 50-nm liposomes composed of an equimolar mixture of POPG and CL. (b) Decrease in (\%) of the $\mathrm{MapZ}_{c y t o}$ resonance intensity for each amide resonance. (c) Graph

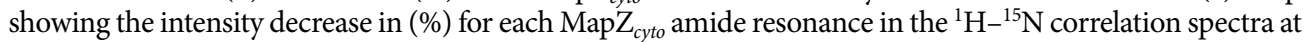
different protein:lipid molar ratios. Data in $(\mathbf{a}-\mathbf{c})$ were collected on a $100 \mu \mathrm{M}$ sample of ${ }^{15} \mathrm{~N} \mathrm{MapZ}_{\text {cyto }}$ in $50 \mathrm{mM}$ Tris, $150 \mathrm{mM} \mathrm{NaCl}$ buffer at $\mathrm{pH}$ 7.5. The stock solution of SUVs was prepared in the same buffer with a $54 \mathrm{mM}$ lipid concentration. Data in $(\mathbf{a}, \mathbf{b})$ corresponds to a 1:62 $\mathrm{MapZ}_{\text {cyto }}$ :lipid molar ratio. NMR experiments were recorded on a 22.4-T spectrometer at $5^{\circ} \mathrm{C}$. Panels (a) through (c) point to an interaction between SUVs and the $\mathrm{N}$-terminal domain of $\mathrm{MapZ}_{\text {cyto }}$ (d) Wide-line (static) ${ }^{2} \mathrm{H}$-ssNMR spectra of PG-d62:CL deuterated multilamellar vesicles (MLVs with a 1:1 molar ratio of both lipids) in the absence (blue) or presence (red) of $\mathrm{MapZ}_{c y t o}$ at a lipid:protein molar ratio of 50:1. (e) Value of the carbon-deuterium order parameters $\left|\mathrm{S}_{\mathrm{CD}}\right|$, obtained from back-calculated ${ }^{2} \mathrm{H}-S S N M R$ spectra of deuterium-labeled phosphoglycerol lipid PG-d62 in the liposomes, in the absence (blue) and in the presence (red) of $\mathrm{MapZ}_{c y t o}$ are plotted as a function of the carbon position along the phosphoglycerol lipid chain. Panels (d,e) evidence a slight reduction of the liposome cohesion.

3 to 11) that may promote the interaction with the negatively charged phosphate groups of the POPG and cardiolipin heads while remaining largely unfolded.

To gain further information on protein-lipid interaction and the associated modifications of membrane structure and dynamics, we performed static ${ }^{2} \mathrm{H}$ solid-state NMR (ssNMR) experiments. The quadrupolar splitting of the ${ }^{2} \mathrm{H}$ nucleus in ${ }^{2} \mathrm{H} 1 \mathrm{D}$ ssNMR spectra directly reports on the phase behaviour of the deuterated lipid and on the 
lipid acyl chain deuterium-carbon order parameter for all deuterated methylene groups of the acyl chain, reflecting the membrane dynamics ${ }^{30,31}$. The so-called De-Pake-ing ${ }^{32}$ decodes the quadrupolar ${ }^{2} \mathrm{H}$ doublet into local order parameter $\left|2 * S_{C D}\right|$ for each deuterium-carbon bond of the methylene groups of the lipid acyl chain. Here we used deuterated 1,2-dipalmitoyl-d62-sn-glycero-3-[phospho-rac-(1-glycerol)] (PG-d62) doped with cardiolipin (CL) (in a 1:1 molar ratio) to reflect the association of $\mathrm{MapZ}_{c y t o}$ on negatively charged lipids. An interaction between membrane-associating proteins with the relevant membrane lipids leads to significant modifications in the dynamics of membrane lipids as reported by the deuterium quadrupolar splitting and the methylene order parameters. Figure 5d shows a slight modification of the quadrupolar splitting pattern of the deuterium signal from all methylene groups of the lipidic acyl chains. This is further evidenced in Fig. 5e after deconvolution of the deuterium signal and calculation of the carbon-deuterium order parameters for each of the methylene groups of the palmitoyl chain. MapZ $\mathrm{Z}_{\text {cyto }}$ influences the membrane structural and dynamical behavior by more significantly lowering the order parameter $\left|2 * \mathrm{~S}_{\mathrm{CD}}\right|$ for the methylene groups that are closer to the polar heads of the lipids (carbon 1 through 9). Discernible effects can nevertheless be perceived up to the inner carbon atoms (carbon 14). The decrease in order parameter reflects the increase in the acyl chain mobility in the liposome, suggesting a fluidifying effect of $\mathrm{MapZ}_{\text {cyto }}$ on PG/CL-containing membranes.

Altogether, monitoring of $\mathrm{MapZ}_{\text {cyto }}$ with liquid-state NMR and deuterated liposomes with ${ }^{2} \mathrm{H} 1 \mathrm{D}$ ssNMR shows that the $\mathrm{N}$-terminal portion of $\mathrm{MapZ}_{\text {cyto }}$ (up to residue 110) interacts with membrane lipids. On one hand, the unfolded and positively charged region of MapZcyto (residues 3 to 11) interacts with the negatively charged lipid heads. On the other hand, the two amphipathic helices (residues 45 to 68 and 79 to 95 ), which present hydrophobic spots surrounded by positively charged and hydrophilic residues, induce packing defects of the lipids by increasing the mobility of their acyl chains ${ }^{33}$. The interaction of the cytoplasmic domain of MapZ with the membrane may be regulated on one side by the membrane composition and curvature and on the other side by the competitive interaction with FtsZ, as the regions of $\mathrm{MapZ}_{c y t o}$ interacting with liposomes and FtsZ overlap. The versatility of this interaction could be essential to the regulatory role of MapZ in the Z-ring positioning.

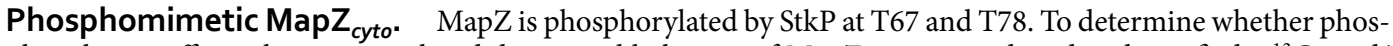
phorylation affects the structural and dynamical behavior of $\mathrm{MapZ}_{\text {cyto }}$, we produced and purified a ${ }^{13} \mathrm{C}-$ and/ or ${ }^{15} \mathrm{~N}$-labeled phosphomimetic mutant of $\mathrm{MapZ}_{\text {cyto }}\left(\mathrm{MapZ}_{\text {cyto }}{ }^{2 T E}\right)$ in which T67 and T78 are replaced with glutamic acid. The ${ }^{1} \mathrm{H}-{ }^{15} \mathrm{~N}$ correlation spectrum of MapZ ${ }_{c y t o}{ }^{2 T E}$ displayed typical features of an unfolded protein and superimposed well with the MapZ $Z_{c y t o}$ spectrum (Fig. 1b) except at the proximity of mutated residues. Backbone resonance assignments were transferred from MapZ ${ }_{c y t o}$ to MapZ $\mathrm{Z}_{c y t o}{ }^{2 T E}$ and completed with a limited set of 3D NMR experiments to the same level of completion as the wild type protein. Analyses of the assigned resonances of $\mathrm{MapZ}_{\text {cyto }}{ }^{2 T E}$, similar to those performed on the wild-type construct, provided a similar secondary structure propensity profile (Fig. S7). This strongly supports the notion that phosphorylation has minimal effect on the structural and dynamic behavior of $\mathrm{MapZ}_{c y t o}$ in the absence of interaction partners and that $\mathrm{MapZ}_{c y t o}{ }^{2 T E}$ remains an intrinsically disordered domain.

To investigate the effect of MapZ phosphorylation on the molecular interaction with FtsZ, we titrated the phophomimetic mutant $\mathrm{MapZ}_{\text {cyto }}{ }_{2 T E}$ with monomeric and polymeric FtsZ. A slightly different protocol was adopted for the sample preparation, i.e. diluted MapZ and FtsZ protein solutions were mixed at the desired molar ratio before the protein mixture was concentrated to a final MapZ concentration of $100 \mu \mathrm{M}$. The same protocol was repeated with wild-type $\mathrm{MapZ}_{\text {cyto }}$ for direct comparison. Titration results are reported in Fig. S8a-c. The regions of $\mathrm{MapZ}_{\text {cyto }}{ }^{2 T E}$ that were the most affected by the monomeric or polymeric forms of FtsZ include a large region spanning residues 21 to 45 and a narrow one centered on residue 81 (Fig. S8a,b). Similar observations were made when the wild-type construct of $\mathrm{MapZ}_{\text {cyto }}$ was mixed with FtsZ (Fig. S8c). Altogether, these results are consistent with the previous observation that phosphorylation does not affect interaction with Fts $Z^{11}$.

We next investigated the interaction of $\mathrm{MapZ}_{c y t o}{ }^{2 T E}$ with liposomes. The profiles of the resonance intensity decrease along the sequence of $\mathrm{MapZ}_{\text {cyto }}$ showed that lipids similarly affected the N-terminal region of the wild-type and 2 TE constructs, with a minimal effect after residue 100 (Fig. S8d,e). Only regions in the vicinity of the mutations displayed a larger peak intensity decrease value than in the corresponding regions of the wild type construct, suggesting that phosphorylation may locally induce a stronger interaction with the SUVs. These in vitro data obtained with the phosphomimetic mutant of $\mathrm{MapZ}_{c y t o}$ are in agreement with previous in vivo studies, which proposed an indirect effect of phosphorylation on cell division since MapZ phosphomimetic and phosphoablative variants retained septal localization and did not alter the Z-ring positioning ${ }^{11,12}$.

\section{Conclusion}

Usually, proteins fold into a unique and stable three-dimensional structure before becoming biologically active. However, studies over the last decade have provided convincing evidence that intrinsically disordered proteins (IDPs) do not adopt a single structure despite being fully functional. High local flexibility of IDPs allows for binding promiscuity and the possibility to interact with many binding partners. The presence of several Molecular Recognition Features (MoRFs) within a sequence of a single IDP allows cells to create molecular protein hubs that serve within cell signaling and regulatory processes ${ }^{34,35}$. We have shown in this study that the cytoplasmic domain of MapZ belongs to this family of intrinsically disordered domains.

In the absence of its natural partners, MapZ $_{\text {cyto }}$ is mainly unstructured except for two specific regions (45-68 and 79-95) that show low but marked propensities to form amphipathic helical structures. Our data suggests that the interaction with FtsZ, which is mainly established by residues 17 to 43 in MapZ, does not affect the structural features of the rest of the protein. This interaction is insensitive to the polymerization status of the FtsZ protein. Consistent with these observations, but in contrast with a recent publication ${ }^{17}$, we did not detect any specific effect of MapZ on FtsZ polymerization or bundling. If MapZ impacts the formation of FtsZ bundles, it might only rely on a variation of local MapZ concentration and crowding, or require the presence of a third yet-to-be identified protein partner. 

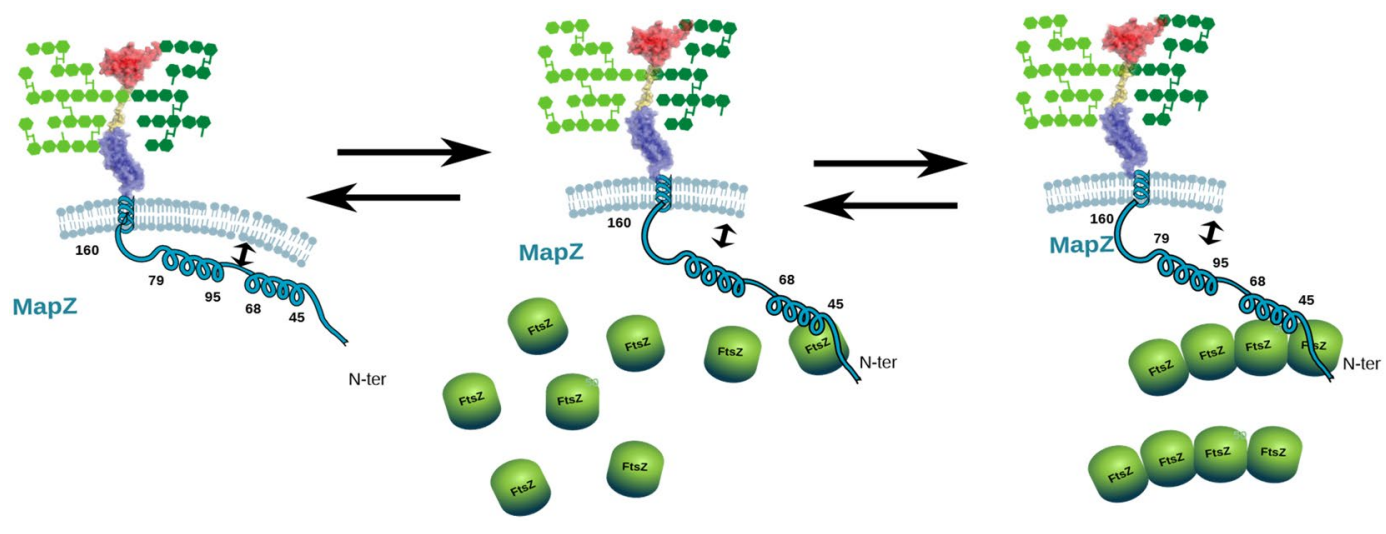

Figure 6. Different states for MapZ and its interaction partners: a complex equilibrium in vivo. The cytoplasmic membrane, the FtsZ monomers, the peptidoglycan, are shown in light blue, as green balls, and as green crosslinked hexagons, respectively. The structural features of MapZ are reported with the ribbon structures of the two extracytoplasmic domains determined by NMR connected with the flexible serine-rich linker in yellow and with the transmembrane and cytoplasmic transient amphipathic helices schematized in blue. Interaction sites and complex formation are visualized through spatial proximity.

In addition, we revealed that MapZ $\mathrm{Z}_{c y t o}$ can interact with the surface of liposomes formed with POPG/PG and cardiolipin mimicking the S. pneumoniae cytoplasmic membrane. Furthermore, the association of MapZ to PG/ $\mathrm{CL}$-containing membranes increases the membrane fluidity. The region of MapZ that interacts with liposomes completely overlaps the region that interacts with FtsZ and extends up to residue 110. As a result, the MapZ-lipid interaction site contains the two identified amphipathic helices transiently forming in the free form, with the first one located in the highly conserved region ranging from residues 18 to 67. Recruitment of FtsZ at mid-cell by the N-terminal region of MapZ could thus be modulated by the availability of this flexible extremity, which is in competition with the membrane for interaction. The outcome of this interaction could be modulated by the curvature or/and the composition of the membrane at mid-cell (Fig. 6). Conversely, MapZ binding to FtsZ may abolish the interaction of MapZ with lipids, thus modifying the fluidity of the membrane at the division site. This change could constitute a step required for cell constriction although the interaction of MapZ with lipids and FtsZ may not be mutually exclusive. Indeed, the region interacting with liposomes only partially overlaps with that of FtsZ, with the region including the two amphipathic helices (residues 45-110) being only involved in lipid binding. In this scenario, the tripartite complex could be an initial step toward the assembly of the divisome. In further support of this idea, it was recently shown that the localization of liquid and gel lipid phases correlates with the localization of FtsZ and the lipid II precursor of $\mathrm{PG}^{36}$.

Finally, we showed that MapZ phosphorylation affects neither its structural organization, nor its interaction with lipids and FtsZ. However, MapZ phosphorylation affects the cell division process ${ }^{11}$. These observations indicate that other partners of MapZ, whose function would be affected by or dependent on MapZ phosphorylation, remain to be identified. Thus, we are still merely at the tip of the iceberg in our understanding of the intricacies of the MapZ system. Complicated as it may be, understanding the molecular dialog occurring at mid-cell between MapZ, FtsZ, lipids and other partners of the divisome is essential to depict the mechanism of cell division in the pneumococcus.

\section{Methods}

Production and purification of MapZ $_{\text {cyto }}$ constructs. The pEtPhos vectors encoding wild-type or phophomimetic T67E/T78E double-mutant of the cytoplasmic domain of MapZ (from residue 1 to 159) followed by a three-amino-acid (LQG) linker, a Tobacco Etch Virus (TEV) protease cleavage site (ENLYFQRGG), and a hexa-histidine tag (adapted from Supplementary Tables 1 and 2 in Fleurie et al. ${ }^{11}$ ) were used to produce $S$. pneumoniae (strain ATCC BAA-255/R6) MapZ $\mathrm{Z}_{\text {cyto }}$ or MapZ $\mathrm{Z}_{c y t o}{ }^{2 T E}$ samples, respectively. ${ }^{15} \mathrm{~N}$ - or ${ }^{13} \mathrm{C},{ }^{15} \mathrm{~N}$-isotopically labeled MapZ $\mathrm{z}_{\text {cyto }}$ and $\mathrm{MapZ}_{\text {cyto }}{ }^{2 T E}$ were expressed in E. coli BL21 (DE3) strain, grown at $37^{\circ} \mathrm{C}$ in M9 medium ${ }^{16}$ supplemented with ${ }^{13} \mathrm{C}$-labeled glucose or/and ${ }^{15} \mathrm{NH}_{4} \mathrm{Cl}$, as well as ampicillin $(100 \mu \mathrm{g} / \mathrm{mL})$. Protein production was induced with $1 \mathrm{mM}$ isopropyl- $\beta$-D-thiogalactopyranoside (IPTG) when the culture reached $\mathrm{OD}_{600}=0.6$. After 3 hours of expression cells were collected and frozen at $-20^{\circ} \mathrm{C}$. For both proteins, the cell pellet was resuspended in $25 \mathrm{~mL}$ of lysis buffer $(50 \mathrm{mM}$ Tris, $500 \mathrm{mM} \mathrm{NaCl}, 10 \mathrm{mM}$ imidazole, $30 \mu \mathrm{g} / \mathrm{mL}$ DNase, $30 \mu \mathrm{g} / \mathrm{mL}$ RNase, 1 tablet of cOmplete protease inhibitor cocktail, $\mathrm{pH} 7.5$ ). After sonication ( $10 \mathrm{~min}$ of $2 \mathrm{~s}$-on-8s-off cycles with an amplification of $60 \%$ on a Branson Ultrasonics apparatus) and centrifugation $\left(46,000 \times \mathrm{g}, 30 \mathrm{~min}, 4^{\circ} \mathrm{C}\right)$, the supernatant was loaded on a Ni-NTA column (Qiagen). Fractions containing the overexpressed protein were pooled and dialyzed overnight at $4{ }^{\circ} \mathrm{C}$ against a $50 \mathrm{mM}$ Tris, $100 \mathrm{mM} \mathrm{NaCl}, \mathrm{pH} 7.5$ buffer. The hexa-histidine tag was cleaved off by incubating with of a solubility-enhanced hexahistidine-tagged L56V/S135G TEV protease ${ }^{37}$ in a 20:1 (w:w) ratio for 3 hours at room temperature. The uncleaved protein and TEV protease were separated from the cleaved protein through immobilized metal affinity chromatography (IMAC) on a Ni-NTA column (Qiagen). The flowthrough containing the cleaved enzyme was concentrated and loaded onto a HiLoad 16/600 Superdex 75 (GE Healthcare) equilibrated in $30 \mathrm{mM}$ HEPES, $200 \mathrm{mM} \mathrm{KCl}$ buffer at pH 7.5 for size exclusion chromatography 
purification. Fractions containing the pure protein were pooled and concentrated. In order to stabilize the proteins, the samples were incubated for $15 \mathrm{~min}$ at $80^{\circ} \mathrm{C}$ before storage. Protein purity was controlled by SDS-PAGE. For long term storage, samples were rapidly frozen in liquid nitrogen and stored in a $-80^{\circ} \mathrm{C}$ freezer.

Production and purification of S. pneumoniae FtsZ. The pMKV18 plasmid, which encodes for the $S$. pneumoniae FtsZ protein without any purification tag was a gift of Dr German Rivas. The corresponding Fts $\mathrm{Z}_{a}$ was expressed and purified as previously described ${ }^{38}$.

The pGEX vector encoding glutathione S-transferase (GST) followed by a short linker (LVPRGS), the TEV protease cleavage site and the wild-type FtsZ from S. pneumoniae was kindly provided by Dr Cecile Morlot. The GST-FtsZ protein was expressed and purified as previously described ${ }^{39}$. In brief, the fusion protein was expressed in E. coli BL21 (DE3) strain, grown at $37^{\circ} \mathrm{C}$ in LB medium supplemented with $100 \mu \mathrm{g} / \mathrm{mL}$ ampicillin. When the culture reached $\mathrm{OD}_{600}=0.7$, the expression was induced with $0.5 \mathrm{mM}$ IPTG and the culture was further incubated overnight at $25^{\circ} \mathrm{C}$. Harvested cells were resuspended in $30 \mathrm{~mL}$ of lysis buffer $(50 \mathrm{mM}$ Tris, $200 \mathrm{mM} \mathrm{NaCl}$, 1 tablet of the cOmplete protease inhibitor cocktail, $\mathrm{pH}$ 8.0). After sonication (10 min of $2 \mathrm{~s}$-on-8s-off cycles with an amplification of $60 \%$ on a Branson Ultrasonics apparatus) and centrifugation $\left(46,000 \times \mathrm{g}, 30 \mathrm{~min}, 4^{\circ} \mathrm{C}\right)$, the supernatant was loaded on a $5 \mathrm{~mL}$ Glutathione Sepharose 4B (GST) affinity column (GE Healthcare, Little Chalfont, United Kingdom). After extensive washing, the GST resin with bound GST-FtsZ was resuspended in $10 \mathrm{~mL}$ of washing buffer ( $50 \mathrm{mM}$ Tris, $200 \mathrm{mM} \mathrm{NaCl}, \mathrm{pH} 8.0)$ and $1 \mathrm{mg}$ of the hexahistidine-tagged L56V/S135G TEV protease ${ }^{37}$ was added. The mixture was stirred for 3 hours at room temperature and the cleaved FtsZ protein was separated from the hexahistidine-tagged TEV on a Ni-NTA column (Qiagen). The fractions in $50 \mathrm{mM}$ Tris, $200 \mathrm{mM} \mathrm{NaCl}$, pH 8.0 containing 0 to $20 \mathrm{mM}$ imidazole were collected, analyzed by gel electrophoresis and concentrated. The final size exclusion chromatography purification step was performed on a HiLoad 16/600 Superdex 200 (GE Healthcare) column equilibrated in $30 \mathrm{mM}$ HEPES, $200 \mathrm{mM} \mathrm{KCl}$ buffer at pH 7.5. Fractions containing pure $\mathrm{Fts}_{b}$ protein were pooled and concentrated and protein purity was controlled by SDS-PAGE.

Fts $Z_{a}$ and Fts $Z_{b}$ only differ in their sequence by the additional glycine scar of the TEV cleavage site at the $\mathrm{N}$-terminus of Fts $\mathrm{Z}_{b}$. To control the correct folding of FtsZ, final protein preparations were systematically checked using ${ }^{1} \mathrm{H} 1 \mathrm{D}$-sculpting NMR experiments ${ }^{40}$ and signal dispersion of the amide and methyl resonances outside of the $[7.5,8.5]$ and $[0.5,-0.5] \mathrm{ppm}$ window, respectively, were ascertained (Fig. S3a). In addition, negative-staining electron microscopy (Fig. S3b) was used to monitor the monomeric versus polymeric state of the FtsZ protein (see electron microscopy subsection below). Polymerization of FtsZ and formation of FtsZ bundles were triggered by the addition of $5 \mathrm{mM}$ guanosine triphosphate (GTP) and $\mathrm{MgCl}_{2}$.

Preparation of liposomes. Unlabeled liposomes for interaction studies with ${ }^{13} \mathrm{C},{ }^{15} \mathrm{~N}$-labeled $\mathrm{MapZ}_{\text {cyto }}$ in liquid-state NMR were prepared as follows. Solutions of the sodium salts of [(2 R)-1[2,3-dihydroxypropoxy(hydroxy)phosphoryl] oxy-3-hexadecanoyloxypropan-2-yl] (Z)octadec9-enoate (1-palmitoyl-2-oleoyl-sn-glycero-3-phospho-(1'-rac-glycerol), POPG, Avanti Polar Lipids Inc.) and [(2 R)-3[[3-[[(2 R)-2,3-di[(9Z)-9-octadecenoyloxy]propoxy]-hydroxyphosphoryl] oxy -2-hydroxypropoxy]-hydroxyphosphoryl] oxy-2-[(9Z)-9-octadecenoyloxy]propyl] (9Z)-9-octadecenoate (1',3'-bis[1,2-dioleoyl-sn-glycero-3-phospho]-glycerol, 18:1 cardiolipin, CL, Avanti Polar Lipids Inc.) in chloroform were mixed together in a 1:1 molar ratio in round-bottomed glass flask. Chloroform was evaporated under a constant nitrogen gas flow until a thin lipid film formed. This dried lipid film was dissolved in an appropriate quantity of $50 \mathrm{mM}$ Tris, $100 \mathrm{mM} \mathrm{NaCl}$ buffer, $\mathrm{pH} 7.5$ for the targeted lipid concentration. The resulting heterogeneous mixture of multilamellar vesicles was homogenized with the Avanti Mini Extruder (Avanti Polar Lipids Inc., Alabaster, USA). The mixture was pressed through a polycarbonate membrane with defined pore diameter, after 10 cycles the membrane was exchanged for a new one with smaller pore size. Membranes with pore sizes of $800,400,200,100$ and $50 \mathrm{~nm}$ were sequentially used to obtain small unilamellar vesicles (SUVs) with diameter of $50 \mathrm{~nm}$. The homogeneity of the final solution and 50-nm size of POPG:CL SUVs were subsequently controlled by diffusion light scattering (DLS) on a Zetasizer Nano S instrument from Malvern Panalytical.

Deuterated liposomes for interaction studies with ${ }^{15} \mathrm{~N}$-labeled $\mathrm{MapZ}_{c y t o}$ in solid-state NMR were prepared from powders of deuterated 1,2-dipalmitoyl-d62-sn-glycero-3-[phospho-rac-(1-glycerol)] (DPPG-d62, Avanti Polar Lipids, Inc.) and unlabeled CL. The two lipids were mixed in a 1:1 molar ratio and dissolved in chloroform. The solvent was evaporated and redissolved as described for unlabelled liposomes and homogenized by three cycles of vortexing, flash freezing in liquid nitrogen for $1 \mathrm{~min}$, and thawing for $10 \mathrm{~min}$ at $40^{\circ} \mathrm{C}$ in a water bath. This protocol generated a milky suspension of micrometer-sized PG-d62:CL multilamellar vesicles (MLVs).

NMR resonance assignments of $\mathrm{MapZ}_{\text {cyto }}$ protein constructs. The $2 \mathrm{D}$ - and $3 \mathrm{D}-\mathrm{NMR}$ experiments were collected on $200 \mu \mathrm{M}^{13} \mathrm{C},{ }^{15} \mathrm{~N}$-labeled MapZ ${ }_{c y t o}$ and $\mathrm{MapZ}_{c y t o}{ }^{2 T E} \mathrm{NMR}$ samples in $30 \mathrm{mM}$ HEPES, $200 \mathrm{mM}$ $\mathrm{KCl}$ buffer at $\mathrm{pH} 7.5$ containing $10 \% \mathrm{D}_{2} \mathrm{O}$. Backbone resonance assignments were carried out using a combination of $2 \mathrm{D}^{1} \mathrm{H}-{ }^{15} \mathrm{~N}-\mathrm{BEST}-\mathrm{TROSY}$ and $3 \mathrm{D} \mathrm{HN}(\mathrm{CO}) \mathrm{CACB}$, iHNCACB, HNCO, HN(CA)CO, H(NCACO)NH experiments. In order to limit the number of overlaps, BEST-TROSY version of the above listed experiments was used ${ }^{24,41}$. For the assignment of side-chains aliphatic carbons, $2 \mathrm{D}^{1} \mathrm{H}_{-}{ }^{13} \mathrm{C}-\mathrm{HSQC}$ and $3 \mathrm{D}(\mathrm{H}) \mathrm{C}(\mathrm{CO}) \mathrm{NH},(\mathrm{H})$ $\mathrm{CCH}-\mathrm{TOCSY}$, and $\mathrm{H}(\mathrm{C}) \mathrm{CHTOCSY}$ experiments were collected. All spectra were recorded at $5^{\circ} \mathrm{C}$ using Bruker AVANCE spectrometers operating at 700 and $850 \mathrm{MHz}$ proton frequency equipped with TCI cryoprobes.

NMR spectra were processed using the TopSpin software by Bruker in its 3.2 version and were analyzed using the CcpNmr Analysis software ${ }^{42}$. The ${ }^{1} \mathrm{H}$ chemical shifts were referenced to the internal standard 4,4-dimethyl-4-silapentane-1 sulfonic acid (DSS) methyl resonance. ${ }^{13} \mathrm{C}$ and ${ }^{15} \mathrm{~N}$ chemical shifts were referenced indirectly using the IUPAC-IUB protocol ${ }^{43}$. Amide, carbonyl, alpha-carbon and beta-carbon resonances could be assigned in $\mathrm{MapZ}_{c y t o}$ with a 95.6\%, 96.4\%, 97.0\%, and 96.9\% completion, respectively. For this construct, 
$73.3 \%$ and $78.3 \%$ of the total aliphatic carbon and proton atoms, respectively, could be assigned, while aromatic side-chains remained too severely overlapped for specific assignment. Backbone resonance assignments were transferred to the phosphomimetic mutant of MapZ, MapZ ${ }_{c y t o}{ }^{2 T E}$, for most resonances. A 3D ${ }^{15} \mathrm{~N}$-TOCSY-HSQC and a $2 \mathrm{D}{ }^{13} \mathrm{C}$-HSQC were used to identify resonances from the E67 and E78 mutated residues and their neighbors. Assignment completion was identical to that of the wild-type protein.

Liquid-state NMR titration experiments. The tendency of FtsZ to polymerize at higher concentrations, even in the absence of GTP, posed the limit on the maximum concentration of the stock solution of FtsZ to be used in titration experiments with monomeric FtsZ (concentration had to be lower than $250 \mu \mathrm{M}$ ). Thus, NMR titration experiments could not be performed in the classical manner by adding a small amount of highly concentrated FtsZ solution into the MapZ NMR sample. Instead, for each MapZ $Z_{c y t o}$ :FtsZ molar ratio, an individual NMR sample was prepared by mixing stock solutions of ${ }^{15} \mathrm{~N}$-labeled $\mathrm{MapZ}_{\text {cyto }}$ and unlabeled FtsZ such that concentration of ${ }^{15} \mathrm{~N}$-labeled $\mathrm{MapZ}_{\text {cyto }}$ was identical over all NMR samples of the titration and in the 30-to-50 $\mu \mathrm{M}$ concentration range. ${ }^{1} \mathrm{H}^{-15} \mathrm{~N}$ BEST-TROSY spectra were then recorded to map changes in $\mathrm{MapZ}_{\text {cyto }}$ signals as induced by the presence of FtsZ. Subsequently, the effect of the FtsZ polymerization state on the interaction with the MapZ $Z_{c y t o}$ was studied. The polymerization of FtsZ was triggered by the addition of $10 \mathrm{mM} \mathrm{GTP}$ and $10 \mathrm{mM} \mathrm{MgCl}_{2}$ (using $100 \mathrm{mM} \mathrm{GTP}$ and $1 \mathrm{M} \mathrm{MgCl}_{2}$ stock solutions) to the NMR samples prepared in the previous step and containing both proteins, FtsZ and MapZ ${ }_{c y t o}$. Samples were then incubated for 5 minutes at room temperature before ${ }^{1} \mathrm{H}_{-}-{ }^{15} \mathrm{~N}$ BEST-TROSY data collection. The same protocol was followed to study the interaction of MapZ ${ }_{c y t o}{ }^{2 T E}$ with monomeric or polymeric FtsZ.

For NMR titration experiments with $50 \mathrm{~nm}$ SUVs, the concentrated aqueous solution of the POPG-CL SUVs ( $54 \mathrm{mM}$ lipid solution in $50 \mathrm{mM}$ Tris, $150 \mathrm{mM} \mathrm{NaCl}$ buffer at $\mathrm{pH} 7.5$ ) was gradually added to the $100 \mu \mathrm{M}^{15} \mathrm{~N}$ MapZ $_{\text {cyto }}$ NMR sample until complete disappearance of several MapZ $Z_{\text {cyto }}$ signals in the ${ }^{1} \mathrm{H}-{ }^{15} \mathrm{~N}$ BEST-TROSY spectra was observed. The same protocol was followed to study the interaction of MapZ ${ }_{c y t o}{ }^{2 T E}$ with 50-nm POPG:CL SUVs.

All ${ }^{1} \mathrm{H}-{ }^{15} \mathrm{~N}$ BEST-TROSY experiments were recorded at $5{ }^{\circ} \mathrm{C}$ using Bruker AVANCE spectrometers equipped with a TCI cryoprobe and operating at 600,700 or $950 \mathrm{MHz}$ proton frequency. Spectra were processed using Bruker Topspin 3.2 and analyzed within CcpNmr Analysis software. Peaks were peaked automatically to determine the optimized peak position and peak intensities were calculated after gaussian lineshape fitting of each $2 \mathrm{D}$-resonance. Resonance intensity decrease values (in \%) were calculated as follows. For each resonance, peak intensity $\mathrm{I}_{0}$ was measured in the reference BEST-TROSY spectrum of $\mathrm{MapZ}_{\text {cyto }}$ in the absence of interactant and peak intensity $I_{1}$ of the same resonance was measured in the BEST-TROSY spectrum of $\mathrm{MapZ}_{c y t o}$ in the presence of interactant. These values were corrected with the dilution factor of $\mathrm{MapZ}_{\text {cyto }}$ in the corresponding experiments and the intensity decrease value in $\%$ was calculated as $100 \times\left(\mathrm{I}_{0}-\mathrm{I}_{1}\right) / \mathrm{I}_{0}$. 3D plots of the titration experiment results were generated using in-house written python scripts and intensity files exported from CcpNmr.

Solid-state NMR studies of $\mathrm{MapZ}_{\text {cyto }}$ interaction with deuterated liposomes. Static solid-state ${ }^{2} \mathrm{H}$ NMR experiments were performed on a Bruker Avance II $500 \mathrm{MHz}$ WB (11.75 T) spectrometer. Samples were equilibrated for $30 \mathrm{~min}$ at $298 \mathrm{~K}$ before data acquisition. ${ }^{2} \mathrm{H}$ NMR experiments on PG-d62 samples were performed at $76 \mathrm{MHz}$ with a phase-cycled quadrupolar echo pulse sequence $\left(90_{x}-\tau\right.$ - $90_{y}-\tau$-acq). Acquisition parameters for ${ }^{2} \mathrm{H}$ spectra were as follows, $500-\mathrm{kHz}$ spectral width, 2.90-ms $\pi / 2$ pulse width, $40-\mathrm{ms}$ inter-pulse $\tau$ delay, and 2-s recycling delay. Spectra were collected with 2048 scans and processed using a 200-Hz Lorentzian line before Fourier transform in the Bruker Topspin 3.2 software. Spectra deconvolution and simulation were applied to determine accurately the experimental quadrupolar splitting. Orientational order parameters $\left(\mathrm{S}_{C D}\right)$ were derived from these values ${ }^{44,45} .{ }^{2} \mathrm{H}$ solid-state NMR spectra were recorded on the micrometer-sized PG-d62:CL multilamellar vesicles (MLVs) in the presence or absence of $\mathrm{MapZ}_{\text {cyto }}$ at a lipid:protein molar ratio of 50:1 for a total lipid concentration of $35 \mathrm{mM}$. For the $\mathrm{MapZ}_{\text {cyto }}$ containing sample, the protein was added to the preformed liposomes and incubated during $30 \mathrm{~min}$ at room temperature.

FtsZ polymerization and bundling assays followed by electron microscopy. To check the polymeric state of the FtsZ samples, the purified FtsZ protein was diluted to $5 \mu \mathrm{M}$ in $30 \mathrm{mM}$ HEPES buffer at $\mathrm{pH}$ 7.6 containing $200 \mathrm{mM} \mathrm{KCl}, 5 \mathrm{mM} \mathrm{MgCl}$ and incubated for 15 minutes at room temperature in the presence or in the absence of $5 \mathrm{mM}$ GTP. For bundling assays of FtsZ, purified recombinant $\mathrm{FtsZ}_{a}$ was diluted to $5 \mu \mathrm{M}$ in $50 \mathrm{mM}$ HEPES buffer at $\mathrm{pH} 7.6$, containing $5 \mathrm{mM} \mathrm{MgCl}_{2}, 200 \mathrm{mM} \mathrm{KCl}, 5 \mathrm{mM} \mathrm{GTP}$, and $8 \%$ (wt/vol) PVA. This sample was incubated for $15 \mathrm{~min}$ at room temperature either in the absence of any protein, in the presence of 5 or $50 \mu \mathrm{M}$ purified recombinant $\mathrm{MapZ}_{c y t o}$, or in the presence of $50 \mu \mathrm{M}$ commercial BSA (Sigma Aldrich) as a control. The BSA powder was resuspended in the $\mathrm{MapZ}_{\text {cyto }}$ buffer (i.e. $30 \mathrm{mM} \mathrm{HEPES,} 200 \mathrm{mM} \mathrm{KCl,} \mathrm{pH} \mathrm{7.5).} \mathrm{In}$ the experiment performed without $\mathrm{MapZ}_{c y t o}$ or BSA, proteins were replaced by an equivalent volume of MapZ $\mathrm{Z}_{c y t o}$ purification buffer. For electron microscopy analysis, negative stain Mica-carbon Flotation Technique (MFT) was used to prepare samples ${ }^{46}$. In brief samples were absorbed on the clean side of a carbon film on mica, stained with $2 \%$ (wt/vol) uranyl acetate. Samples were then transferred to a 400 -mesh copper grid, which was subsequently air-dried. Images were taken under low dose conditions $\left(<10 \mathrm{e}^{-} / \AA^{2}\right)$ with defocus values between 1.2 and $2.5 \mu \mathrm{m}$ on a Technai $12 \mathrm{FEI} \mathrm{LaB6}$ electron microscope at $120 \mathrm{kV}$ accelerating voltage. Image acquisition was performed with calibrated nominal magnifications ranging from 440 to 23,000, using a CCD Gatan ORIUS SC1000 camera (Gatan, Inc., Pleasanton, CA).

Received: 13 November 2019; Accepted: 13 February 2020;

Published online: 04 March 2020 


\section{References}

1. Adams, D. W. \& Errington, J. Bacterial cell division: assembly, maintenance and disassembly of the Z ring. Nat. reviews. Microbiol. 7, 642-53, https://doi.org/10.1038/nrmicro2198 (2009).

2. Du, S. \& Lutkenhaus, J. At the Heart of Bacterial Cytokinesis: The Z Ring. Trends Microbiol. 27, 781-791, https://doi.org/10.1016/j. tim.2019.04.011 (2019).

3. Mateos-Gil, P., Tarazona, P. \& Vélez, M. Bacterial cell division: modeling FtsZ assembly and force generation from single filament experimental data. FEMS microbiology reviews 43, 73-87, https://doi.org/10.1093/femsre/fuy039 (2019).

4. Wu, L. J. \& Errington, J. Nucleoid occlusion and bacterial cell division. Nat. Rev. Microbiol. 10, 8-12, https://doi.org/10.1038/ nrmicro2671 (2012).

5. Rowlett, V. W. \& Margolin, W. The bacterial Min system. Curr. Biol. 23, R553-R556, https://doi.org/10.1016/j.cub.2013.05.024 (2013).

6. Eswara, P. J. \& Ramamurthi, K. S. Bacterial Cell Division: Nonmodels Poised to Take the Spotlight. Annu. Rev. Microbiol. 71, 393-411, https://doi.org/10.1146/annurev-micro-102215-095657 (2017).

7. Thanbichler, M. \& Shapiro, L. MipZ, a Spatial Regulator Coordinating Chromosome Segregation with Cell Division in Caulobacter. Cell 126, 147-162, https://doi.org/10.1016/j.cell.2006.05.038 (2006).

8. Willemse, J., Borst, J. W., De Waal, E., Bisseling, T. \& Van Wezel, G. P. Positive control of cell division: FtsZ is recruited by SsgB during sporulation of Streptomyces. Genes Dev. 25, 89-99, https://doi.org/10.1101/gad.600211 (2011).

9. Treuner-Lange, A. et al. PomZ, a ParA-like protein, regulates Z-ring formation and cell division in Myxococcus xanthus. Mol. Microbiol. 87, 235-253, https://doi.org/10.1111/mmi.12094 (2013).

10. van Raaphorst, R., Kjos, M. \& Veening, J.-W. Chromosome segregation drives division site selection in Streptococcus pneumoniae. Proc. Natl. Acad. Sci. 114, E5959-E5968, https://doi.org/10.1073/pnas.1620608114 (2017).

11. Fleurie, A. et al. MapZ marks the division sites and positions FtsZ rings in Streptococcus pneumoniae, Nature 516, 259-262, https:// doi.org/10.1038/nature13966 (2014).

12. Holecková, N. et al. LocZ is a new cell division protein involved in proper septum placement in Streptococcus pneumoniae. mBio, https://doi.org/10.1128/mBio.01700-14 (2014).

13. Vollmer, W., Massidda, O. \& Tomasz, A. The Cell Wall of Streptococcus pneumoniae. Microbiol. Spectr. 7, https://doi.org/10.1128/ microbiolspec.GPP3-0018-2018 (2019).

14. Garcia, P. S., Simorre, J.-P., Brochier-Armanet, C. \& Grangeasse, C. Cell division of Streptococcus pneumoniae: think positive! Curr. Opin. Microbiol. 34, 18-23, https://doi.org/10.1016/J.MIB.2016.07.014 (2016).

15. Li, Y. et al. MapZ Forms a Stable Ring Structure That Acts As a Nanotrack for FtsZ Treadmilling in Streptococcus mutans. ACS Nano 12, 6137-6146, https://doi.org/10.1021/acsnano.8b02469 (2018).

16. Manuse, S. et al. Structure-function analysis of the extracellular domain of the pneumococcal cell division site positioning protein MapZ. Nat. Commun. 7, https://doi.org/10.1038/ncomms12071 (2016).

17. Feng, Z. et al. Multi-functional regulator MapZ controls both positioning and timing of FtsZ polymerization. Biochem. J. 476, 1433-1444, https://doi.org/10.1042/BCJ20190138 (2019).

18. Manuse, S., Fleurie, A., Zucchini, L., Lesterlin, C. \& Grangeasse, C. Role of eukaryotic-like serine/threonine kinases in bacterial cell division and morphogenesis. FEMS Microbiol. Rev. 40, 41-56, https://doi.org/10.1093/femsre/fuv041 (2016).

19. Dosztanyi, Z., Csizmok, V., Tompa, P. \& Simon, I. IUPred: web server for the prediction of intrinsically unstructured regions of proteins based on estimated energy content. Bioinformatics 21, 3433-3434, https://doi.org/10.1093/bioinformatics/bti541 (2005).

20. Radivojac, P. et al. Intrinsic disorder and functional proteomics. Biophys. journal 92, 1439-56, https://doi.org/10.1529/ biophys.106.094045 (2007).

21. Vacic, V., $\dagger$ et al. Characterization of Molecular Recognition Features, MoRFs, and their binding partners, J. Proteom. Res. 6, 23512366, https://doi.org/10.1021/PR0701411 (2007).

22. Tompa, P. \& Fuxreiter, M. Fuzzy complexes: polymorphism and structural disorder in protein-protein interactions. TRENDS Biochem. Sci. 33, 2-8, https://doi.org/10.1016/j.tibs.2007.10.003 (2008).

23. Ashkenazy, H. et al. ConSurf 2016: an improved methodology to estimate and visualize evolutionary conservation in macromolecules. Nucleic Acids Res. 44, W344-W350, https://doi.org/10.1093/nar/gkw408 (2016).

24. Brutscher, B. et al. NMR Methods for the study of instrinsically disordered proteins structure, dynamics, and interactions: General overview and practical guidelines. 49-122, https://doi.org/10.1007/978-3-319-20164-1_3 (in Adv. Exp.Med. Biol. series, Springer, Cham, 2015).

25. Tamiola, K., Acar, B. \& Mulder, F. A. A. Sequence-specific random coil chemical shifts of intrinsically disordered proteins. J. Am. Chem. Soc. 132, 18000-18003, https://doi.org/10.1021/ja105656t (2010).

26. Tamiola, K., Mulder, F. \& Using, N. M. R. chemical shifts to calculate the propensity for structural order and disorder in proteins. Biochem. Soc. Transactions 40, 1014-1020, https://doi.org/10.1042/bst20120171 (2012).

27. Gautier, R., Douguet, D., Antonny, B. \& Drin, G. HELIQUEST: a web server to screen sequences with specific helical properties. Bioinformatics 24, 2101-2102, https://doi.org/10.1093/bioinformatics/btn392 (2008).

28. Abyzov, A. et al. Identification of dynamic modes in an intrinsically disordered protein using temperature-dependent NMR relaxation. J. Am. Chem. Soc. 138, 6240-6251, https://doi.org/10.1021/jacs.6b02424 (2016).

29. Trombe, M.-C., Lanéelle, M.-A. \& Lanéelle, G. Lipid composition of aminopterin-resistant and sensitive strains of Streptococcus pneumoniae. Effect of aminopterin inhibition. Biochimica et Biophys. Acta 574, 290-300, https://doi.org/10.1016/00052760(79)90010-9 (1979).

30. Dufourc, E. J., Parish, E. J., Chitrakorn, S. \& Smith, I. C. P. Structural and dynamical details of cholesterol-lipid interaction as revealed by deuterium NMR. Biochemistry 23, 6062-6071, https://doi.org/10.1021/bi00320a025 (1984).

31. Petrache, H. I., Dodd, S. W. \& Brown, M. F. Area per lipid and acyl length distributions in fluid phosphatidylcholines determined by ${ }^{2}$ H NMR Spectroscopy. Biophys. J. 79, 3172-3192, https://doi.org/10.1016/S0006-3495(00)76551-9 (2000).

32. Davis, J. H. The description of membrane lipid conformation, order and dynamics by ${ }^{2} \mathrm{H}-\mathrm{NMR}$. Biochimica et Biophys. Acta 737 , 117-171, https://doi.org/10.1016/0304-4157(83)90015-1 (1983).

33. Das, T. \& Eliezer, D. Membrane interactions of intrinsically disordered proteins: The example of alpha-synuclein. Biochimica et Biophys. Acta - Proteins Proteomics 1867, 879-889, https://doi.org/10.1016/j.bbapap.2019.05.001 (2019).

34. Dunker, A. K., Cortese, M. S., Romero, P., Iakoucheva, L. M. \& Uversky, V. N. Flexible nets. The roles of intrinsic disorder in protein interaction networks. The FEBS J. 272, 5129-5148, https://doi.org/10.1111/j.1742-4658.2005.04948.x (2005).

35. Dosztányi, Z., Chen, J., Dunker, A. K., Simon, I. \& Tompa, P. Disorder and sequence repeats in Hub Proteins and their implications for network evolution. J. Proteome Res. 5, 2985-2995, https://doi.org/10.1021/pr060171o (2006).

36. Calvez, P., Jouhet, J., Vié, V., Durmort, C. \& Zapun, A. Lipid phases and cell geometry during the cell cycle of Streptococcus pneumoniae. Front. Microbiol. 10, 351, https://doi.org/10.3389/fmicb.2019.00351 (2019).

37. Cabrita, L. D. et al. Enhancing the stability and solubility of TEV protease using in silico design. Protein Sci. 16, 2360-2367, https:// doi.org/10.1110/ps.072822507 (2007).

38. Salvarelli, E. et al. The cell division protein FtsZ from Streptococcus pneumoniae exhibits a GTPase activity delay. J. Biol. Chem. 290, 25081-25089, https://doi.org/10.1074/jbc.M115.650077 (2015).

39. Jacq, M. et al. Remodeling of the Z-Ring nanostructure during the Streptococcus pneumoniae cell cycle revealed by photoactivated localization microscopy. mBio 6, e01108-15, https://doi.org/10.1128/mBio.01108-15 (2015). 
40. Hwang, T.-L. \& Shaka, A. Water suppression that works. Excitation sculpting using arbitrary waveforms and pulsed field gradients. J. Magn. Reson. Ser. A 112, 275-279 (1995).

41. Solyom, Z. et al. BEST-TROSY experiments for time-efficient sequential resonance assignment of large disordered proteins. J. Biomol. NMR 55, 311-321, https://doi.org/10.1007/s10858-013-9715-0 (2013).

42. Vranken, W. F. et al. The CCPN data model for NMR spectroscopy: Development of a software pipeline. Proteins-Structure Funct. Bioinforma. 59, 687-696 (2005).

43. Markley, J. L. et al. Recommendations for the presentation of NMR structures of proteins and nucleic acids. IUPACIUBMB-IUPAB inter-union task group on the standardization of data bases of protein and nucleic acid structures determined by NMR spectroscopy. Eur. J. Biochem. 256, 1-15, https://doi.org/10.1046/j.1432-1327.1998.2560001.x (1998).

44. Seelig, J. Deuterium magnetic resonance: theory and application to lipid membranes. Q. reviews biophysics 10, 353-418 (1977).

45. Vogel, A. et al. Lipid modifications of a ras peptide exhibit altered packing and mobility versus host membrane as detected by $2 \mathrm{H}$ solid-state NMR. J. Am. Chem. Soc. 127, 12263-12272, https://doi.org/10.1021/ja051856c (2005).

46. Scarff, C. A., Fuller, M. J. G., Thompson, R. F. \& Iadaza, M. G. Variations on Negative Stain Electron Microscopy Methods: Tools for Tackling Challenging Systems. J. Vis. Exp. 1-8, https://doi.org/10.3791/57199 (2018).

\section{Acknowledgements}

Authors thank Bernhard Brutscher, Nathanael Caveney and Waldemar Vollmer for careful reading of the manuscript. C.G., C.M.B., and J-P.S. were supported by the ANR grant (ANR-15-CE32-0001), which also funded T.H. post-doctoral fellowship. C.G. and C. M. were supported by the ANR grant (ANR-16-CE11-0016). Financial support from the IR-RMN-THC Fr3050 CNRS for conducting the research is gratefully acknowledged. The authors acknowledge the platforms of the Grenoble Instruct-ERIC center (ISBG; UMS 3518 CNRS-CEAUGA-EMBL) within the Grenoble Partnership for Structural Biology (PSB). Platform access was supported by FRISBI (ANR-10-INBS-05-02) and GRAL, a project of the University Grenoble Alpes graduate school (Ecoles Universitaires de Recherche) CBH-EUR-GS (ANR-17-EURE-0003). BH is supported by CNRS Momentum and the Region Nouvelle Aquitaine for a post-doctoral funding.

\section{Author contributions}

T.H. conducted most of the sample preparation and NMR experiments. D.F. and C.M. performed and analyzed all of the electron microscopy experiments. I.A., J.-P.L. and M.R. prepared protein samples. D.M. and B.H. performed and analyzed the static solid-state experiments on deuterated lipids. J.-P.S., C.G. and C.M.B. conceived the experiment(s) and coordinated the writing of the manuscript. All authors reviewed the manuscript.

\section{Competing interests}

The chemical shifts for $\mathrm{MapZ}_{c y t o}$ and $\mathrm{MapZ}_{\text {cyto }}{ }^{2 T E}$ were deposited in the Biological Magnetic Resonance Bank under the accession numbers 28006 and 28005 , respectively. The authors declare no competing interest. The corresponding author is responsible for submitting a competing interests statement on behalf of all authors of the paper.

\section{Additional information}

Supplementary information is available for this paper at https://doi.org/10.1038/s41598-020-61036-9.

Correspondence and requests for materials should be addressed to C.G. or J.-P.S.

Reprints and permissions information is available at www.nature.com/reprints.

Publisher's note Springer Nature remains neutral with regard to jurisdictional claims in published maps and institutional affiliations.

Open Access This article is licensed under a Creative Commons Attribution 4.0 International License, which permits use, sharing, adaptation, distribution and reproduction in any medium or format, as long as you give appropriate credit to the original author(s) and the source, provide a link to the Creative Commons license, and indicate if changes were made. The images or other third party material in this article are included in the article's Creative Commons license, unless indicated otherwise in a credit line to the material. If material is not included in the article's Creative Commons license and your intended use is not permitted by statutory regulation or exceeds the permitted use, you will need to obtain permission directly from the copyright holder. To view a copy of this license, visit http://creativecommons.org/licenses/by/4.0/.

(c) The Author(s) 2020 